\title{
A Generalized Recursive Coordinate Reduction Method for Multibody System Dynamics
}

\author{
J. H. Critchley \& K. S. Anderson \\ Department of Mechanical, Aeronautical, and Nuclear Engineering, \\ Rensselaer Polytechnic Institute, Troy, New York, USA
}

\begin{abstract}
The method of recursive coordinate reduction (RCR) offers solutions to the forward problem of multibody dynamics at a cost in which the number of operations is linear in both the number of generalized coordinates, $n$, and the number of independent algebraic constraints, $m(e . g ., O(n+m))$. However, the RCR is presently restricted in applicability (albeit broad) and susceptible to formulation singularities. This article develops two methods for avoiding formulation singularities as well as a recursive general coupled loop solution that extends the RCR to the complete set of multibody systems. Application of these techniques are further illustrated with a special five-bar linkage. The existing RCR coupled with these developments constitute a generalized recursive coordinate reduction method that should be used in place of the traditional "O(n)" constraint technique (truly $\left.O\left(n+n m^{2}+m^{3}\right)\right)$ for superior $O(n+m)$ computational performance.
\end{abstract}

Address all correspondence to J. H. Critchley; james@multibodydynamics.com. 


\section{NOMENCLATURE}

\begin{tabular}{|c|c|}
\hline$F_{\mathcal{A}}{ }^{k}$ & The spatial acceleration vector of body $k$ as measured in frame $F$ \\
\hline$F \hat{\mathcal{A}}^{k}$ & $\begin{array}{l}\text { The recursive spatial vector of acceleration components associated with body } k \text { measured in frame } F \text { which are } \\
\left.\text { explicit in the system generalized speed derivatives ( } \dot{u}^{\prime} s\right)\end{array}$ \\
\hline$F \mathcal{A}_{t}^{k}$ & $\begin{array}{l}\text { The spatial vector of acceleration components associated with body } k \text { measured in frame } F \text { which are not explicit } \\
\text { in the system generalized speeds (u's) }\end{array}$ \\
\hline$A n[k]$ & The ancestral body set of a body $k$ \\
\hline$b$ & Index corresponding to the branching body in a coupled loop system \\
\hline$c h[k]$ & The set of child bodies of a body $k$ \\
\hline$D i$ & An arbitrary dependent coordinate on local loop $i$ \\
\hline$d e p_{i}$ & The set of dependent coordinates on local loop $i$ \\
\hline $\operatorname{Des}[k]$ & The descendant body set of a body $k$ \\
\hline $\mathcal{F}_{k}$ & The spatial force vector of body $k$ \\
\hline$\hat{\mathcal{F}}_{k}$ & The recursive spatial force vector of body $k$ \\
\hline$\hat{\mathcal{F}}_{M}$ & A column matrix of the recursive spatial force vectors $\hat{\mathcal{F}}^{k_{i}}$ with $k_{i} \in$ independents $f_{i}$ through $l_{i}$ \\
\hline$\stackrel{\vee}{\mathcal{F}}^{k} k_{i}$ & $\begin{array}{l}\text { The spatial force vector associated with independent generalized speed } u_{k i} \text { in the coordinate reduced equation } \\
\text { of motion on local loop } i\end{array}$ \\
\hline$\breve{\mathcal{F}}^{k_{i}}$ & A spatial force vector intermediate quantity associated with body $k$ \\
\hline$f_{i}$ & A context dependent "first" coordinate associated with a local loop $i$ \\
\hline $\mathcal{G}^{k_{i}}$ & $\begin{array}{l}\text { A row matrix of spatial tensors representing the dependence of spatial velocity } k_{i} \text { on outboard independent } \\
\text { generalized speeds }\end{array}$ \\
\hline $\mathcal{H}^{k_{i}}$ & $\begin{array}{l}\text { A row matrix of spatial tensors representing the dependence on outboard independent generalized speeds in } \\
\text { spatial velocity } k_{i}^{\prime} \text { s expression in terms of } p_{i}\end{array}$ \\
\hline $\mathcal{H}_{r}^{k_{i}}$ & The spatial tensor element of $H^{k_{i}}$ associated with independent generalized speed $r$ \\
\hline$\hat{\mathcal{H}}^{k_{i}}$ & $\begin{array}{l}\text { A row matrix of spatial tensors representing the dependence on outboard independent generalized speeds in } \\
\text { the } k_{i}^{\text {th }} \text { coordinate reduced equation of motion (outboard of } p_{i} \text { ) }\end{array}$ \\
\hline$\breve{\mathcal{H}}^{k_{i}}$ & A row matrix of spatial tensor intermediate quantities \\
\hline$I_{i}$ & An arbitrary independent coordinate on local loop $i$ \\
\hline $\mathcal{I} k$ & The spatial inertia tensor of body $k$ \\
\hline$\hat{\mathcal{I}}^{k}$ & The recursive spatial inertia tensor of body $k$ \\
\hline${ }^{0_{i}} \widehat{\mathcal{I}}^{k_{i}}$ & $\begin{array}{l}\text { A recursive spatial inertia tensor associated with independent generalized speed } u_{k_{i}} \text { in the coordinate reduced } \\
\text { equation of motion on local loop } i\end{array}$ \\
\hline$\widehat{\mathcal{I}}^{k_{i} ; 0_{i}}$ & $\begin{array}{l}\text { A recursive spatial inertia tensor associated with independent generalized speed } u_{k} \text { in the coordinate reduced } \\
\text { equation of motion on local loop } i\end{array}$ \\
\hline${ }^{0_{i}} \widehat{\mathcal{I}}^{k ; 0_{j}}$ & $\begin{array}{l}\text { A recursive spatial inertia tensor associated with independent generalized speed } u_{k} \text { in the coordinate reduced } \\
\text { equation of motion existing only on common bodies of two coupled loops } i \text { and } j\end{array}$ \\
\hline$\widehat{\mathcal{I}}_{M}^{0_{i}}$ & A column matrix of the recursive spatial inertia tensors $\widehat{\mathcal{I}}^{k_{i} ; 0_{i}}$ with $k_{i} \in$ independents $f_{i}$ through $l_{i}$ \\
\hline$\widehat{\mathcal{I}}_{M}^{p_{i}}$ & A column matrix of the recursive spatial inertia tensors ${ }^{0_{i}} \breve{\mathcal{I}}^{k_{i}}$ with $k_{i} \in$ independents $f_{i}$ through $l_{i}$ \\
\hline${ }^{0_{i}} \breve{\mathcal{I}}^{k_{i}}$ & $\begin{array}{l}\text { A spatial inertia tensor associated with independent generalized speed } u_{k_{i}} \text { in the coordinate reduced equation of } \\
\text { motion on local loop } i\end{array}$ \\
\hline$\breve{\mathcal{I}}^{k_{i} ; 0_{i}}$ & $\begin{array}{l}\text { A spatial inertia tensor associated with independent generalized speed } u_{k_{i}} \text { in the coordinate reduced equation of } \\
\text { motion on local loop } i\end{array}$ \\
\hline${ }^{0_{i}} \breve{I}^{k_{i}}$ & A spatial inertia tensor intermediate quantity associated with independent generalized speed $u_{k_{i}}$ \\
\hline
\end{tabular}




\begin{tabular}{|c|c|}
\hline$\breve{\mathcal{I}}^{k_{i} ; 0_{i}}$ & A spatial inertia tensor intermediate quantity associated with independent generalized speed $u_{k_{i}}$ \\
\hline$\stackrel{\overrightarrow{\vec{I}}}{ }^{B / B^{*}}$ & The central inertia dyadic of body $B$ \\
\hline$i$ & Index corresponding to an arbitrary kinematic loop \\
\hline$i c h[k]$ & The body set of independent children of body $k$ \\
\hline$i D e s[k]$ & The body set of independent descendants of body $k$ \\
\hline ind $_{i}$ & The set of independent coordinates on local loop $i$ \\
\hline$j$ & Index corresponding to an arbitrary body, coordinate, or kinematic loop \\
\hline$j_{i}$ & Index corresponding to an arbitrary body or coordinate number on local loop $i$ \\
\hline $\mathcal{K}_{i}$ & $\begin{array}{l}\text { A diagonal matrix of the spatial partial velocity vectors associated with independent generalized speeds } f_{i} \\
\text { through } l i\end{array}$ \\
\hline$k$ & Index corresponding to an arbitrary body or coordinate number \\
\hline$k_{i}$ & Index corresponding to an arbitrary body or coordinate number on local loop $i$ \\
\hline$l_{i}$ & The "last" independent coordinate associated with a local loop $i$ \\
\hline$N$ & The Newtonian (or inertial) reference frame \\
\hline$n$ & The number of generalized coordinates in a multibody system \\
\hline$n_{I}$ & The number of independent coordinates outboard of the virtual terminal body $p_{i}$ on local loop $i$ \\
\hline$n_{i}$ & The number of generalized coordinates in a local kinematic loop $i$ \\
\hline$\widehat{\mathcal{M}}^{k}$ & The recursive triangularized mass matrix diagonal associated with generalized speed $k$ \\
\hline$\widehat{\mathcal{M}}_{M}$ & $\begin{array}{l}\text { A square matrix of the coupled block triangularized mass matrix terms which are } \hat{\mathcal{M}}^{k_{i}} \text { with } k_{i} \in \text { independents } f_{i} \\
\text { through } l_{i}\end{array}$ \\
\hline$m$ & The number of independent algebraic constraint relations in a multibody system \\
\hline$m_{i}$ & The number of independent algebraic constraint relations in the local loop i \\
\hline$m_{B}$ & The mass of a body $B$ \\
\hline $\mathcal{P}_{r}^{k}$ & The spatial partial velocity vector representing the partial derivative of $N \mathcal{V}^{k}$ with respect to $u_{r}$ \\
\hline$\tilde{\mathcal{P}}_{\mathrm{r}}^{k}$ & $\begin{array}{l}\text { The constrained spatial partial velocity explicitly accounting for the constraint through the direct embedding of } \\
\text { the constraints in the equations on motion }\end{array}$ \\
\hline$p_{i}$ & Index corresponding to the virtual terminal body of the local loop $i$ \\
\hline$p[k]$ & The parent of a body $k$ \\
\hline $\mathcal{Q}_{k i}$ & A scalar intermediate quantity associated with the local kinematics of loop $i$ and body $k$ \\
\hline $\mathcal{R}_{k i}$ & A spatial tensor intermediate quantity associated with the local kinematics of loop $i$ and body $k$ \\
\hline$r$ & Index corresponding to an arbitrary body or coordinate number \\
\hline $\mathcal{S}^{k}$ & $\begin{array}{l}\text { The spatial shift tensor which represents the position vector cross product coupling of linear and angular } \\
\text { relationships associated with body } k \text { and it's parent body }\end{array}$ \\
\hline$r \mathcal{S}^{k}$ & $\begin{array}{l}\text { The spatial shift tensor which represents the position vector cross product coupling of linear and angular } \\
\text { relationships between body } k \text { and body } r\end{array}$ \\
\hline $\mathcal{T}^{k}$ & The spatial triangularization tensor associated with generalized speed $k$ \\
\hline $\mathcal{U}$ & The spatial unity tensor \\
\hline $\overrightarrow{\vec{U}}$ & A unity dyadic \\
\hline$u_{k}$ & The generalized speed associated with the motion of body $k$ relative to it's parent body \\
\hline$u_{\mathrm{i}}$ & A matrix of the independent generalized speeds $f_{i}$ through $I_{i}$ \\
\hline$u_{k}$ & The generalized acceleration associated with the motion of body $k$ relative to it's parent body \\
\hline
\end{tabular}




\begin{tabular}{|c|c|}
\hline$F \mathcal{V}^{k}$ & The spatial velocity vector of body $k$ as measured in frame $F$ \\
\hline$F \hat{\mathcal{V}}^{k}$ & $\begin{array}{l}\text { The recursive spatial vector of velocity components associated with body } k \text { measured in frame } F \text { which are } \\
\text { explicit in the system generalized speeds ( } u^{\prime} s \text { ) }\end{array}$ \\
\hline$F \mathcal{V}_{t}^{k}$ & $\begin{array}{l}\text { The spatial vector of velocity components associated with body } k \text { measured in frame } F \text { which are not explicit in } \\
\left.\text { the system generalized speeds ( } u^{\prime} s\right)\end{array}$ \\
\hline${ }^{F} \vec{v}^{B^{*}}$ & The velocity of a body B's center of mass as measured in frame $F$ \\
\hline $\mathcal{W}^{k_{i}}$ & $\begin{array}{l}\text { A spatial tensor intermediate quantity recursively relating the kinematics of body } k_{i}, p\left[k_{i}\right] \text {, and } n_{i} \text { through the } \\
\text { direct embedding of constraints on loop } i\end{array}$ \\
\hline $\mathcal{X}^{k i}$ & $\begin{array}{l}\text { A spatial tensor intermediate quantity recursively relating the kinematics of body } k_{i}, p\left[k_{i}\right] \text {, and } n_{i} \text { through the } \\
\text { direct embedding of constraints on loop } i\end{array}$ \\
\hline $\mathcal{Y}^{k_{i}}$ & A spatial tensor relating the kinematics of body $k_{i}, p_{i}$, and $n_{i}$ through direct embedding of constraints on loop $i$ \\
\hline $\mathcal{Z}^{k_{i}}$ & A spatial tensor relating the kinematics of body $k_{i}, p_{i}$, and $n_{i}$ through direct embedding of constraints on loop $i$ \\
\hline $0_{i}$ & The base body of loop $i$ which branches to form the loop \\
\hline${ }^{F} \vec{\omega}^{B}$ & The angular velocity of body $b$ as measured in frame $f$ \\
\hline
\end{tabular}

\section{INTRODUCTION}

The complexity of a multibody system model is often simplified to obtain solutions in a desired (or realistic) time scale for applications such as realtime operator or hardware-in-the-loop simulation, robotics and control simulation, model-based predictive control, virtual prototyping, design optimization, and the like. The fidelity of such applications is most generally compromised by both the model reduction and simplification required to realize simulation/analysis time constraints. In this respect, rapid computation of the forward dynamics of multibody systems has an important role in many areas. This has prompted researchers whose interests lie in a wide variety of fields to investigate methods for increasing the performance of multibody forward dynamics methods, which had traditionally computed solutions for the $n$ independent coordinate accelerations in order $n^{3}$ $\left(O\left(n^{3}\right)\right)$ operations overall.

In 1983, Featherstone [1] presented an efficient formulation of existing $O(n)$ methods (those of Vereshegin [2] and Armstrong [3]), called the articulated body algorithm (ABA). Based on Walker and Orin's efficient $O\left(n^{3}\right)$ forward solutions [4], which were in turn derived from recursive $O(n)$ solutions of the inverse problem $[5,6]$, the ABA achieved linear cost versus complexity $(O(n))$ for the forward problem through the exploitation of additional recursive relationships.
Since the ABA's introduction, myriad $O(n)$ methods that extend the applicability and flexibility of the recursive formulation have followed. Most notably are the extensions involving various classes of flexible bodies [7-9], integration with optimal filtering and control $[10,11]$, design sensitivity $[12,13]$, and parallel computing [14-17]. Suffice it to say that improvements to the underlying recursive $O(n)$ formulation benefit all of these areas.

The first recursive $O(n)$ algorithms could be applied only to tree-type multibody systems but were quickly extended by Featherstone [18] and Bae and Haug [19] to include those with kinematic (closed) loops. However, these and most other subsequent closed loop solutions require the formation and inversion of an $m \times m$ constraint matrix, involving $O\left(\mathrm{~nm}^{2}\right)$ and $O\left(\mathrm{~m}^{3}\right)$ operations, respectively (where $m$ is the number of independent algebraic constraints). Because of the cubic nature of the constraint solution, these "order $n$ " algorithms can be outperformed by efficient traditional $O\left(n^{3}\right)$ methods for modest to heavily constrained systems.

As an alternative to existing $O(n)$ closed-loop methods, Valášek [20] outlined two methods for the treatment of multibody loop systems, in which the effects of dependent loop coordinates could be obtained in terms of the independent loop coordinates in a number of computations linear in the number of dependent coordinates. In an independent development, Anderson 
[21] formulated similar, though more general, equations based on his $O(n)$ algorithm, which implements Kane's method [22]. Anderson espoused the potential benefits of such a method, which, apart from offering the improved computational complexity cited by Valášek, also included superior constraint stability relative to the traditional " $O(n)$ " constraint formulation.

More recently, the authors [23] have extended the formulation, now referred to as recursive coordinate reduction (RCR), to include a broad array of heavily coupled multiloop systems. This work directly demonstrates the predicted results of Anderson [21] and details the limitations of the formulation.

This article presents modifications to the RCR for the limiting cases of Anderson and Critchley [23], which pertain to arbitrary selection of dependent coordinates and coupled loops with different loop base bodies. Surpassing these limitations results in a generalized recursive coordinate reduction method capable of accommodating arbitrary multibody systems. Indeed, this approach is now being applied by the authors to model systems with such different applications (as well as spatial and temporal scales) as automotive drivelines and suspensions, microelectromechanical systems (MEMS) and aspects of their manufacturing/assembly processes, and detailed modeling of the dynamic behavior and material characteristics of nanostructures and molecular systems.

\section{PRELIMINARIES}

In this section, specialized multibody notations are introduced and used to illustrate the recursive relationships and procedures fundamental to the $O(n)$ algorithms, which will aid in the subsequent developments.

\subsection{Spatial Tensor Notation}

A spatial tensor notation has been adopted to greatly simplify the form of the equations used to describe properties of multibody systems. Spatial tensors are linear algebraically augmented three-dimensional tensors (vectors and dyadics) used to combine translational and rotational information into a common uppercase script symbol. For example, a spatial velocity tensor $\mathcal{V}$ and a spatial inertia tensor $\mathcal{I}$ may be given as

$$
\begin{aligned}
& { }^{N} \mathcal{V}^{B}=\left[\begin{array}{c}
{ }^{N} \vec{\omega}^{B} \\
{ }^{N} \vec{v}^{B}
\end{array}\right] \\
& \mathcal{I}^{B}=\left[\begin{array}{cc}
{ }^{B} \overrightarrow{\vec{I}}^{B / B^{*}} & 0 \\
0 & m_{B}
\end{array}\right]=\left[\begin{array}{cc}
{ }^{B} \overrightarrow{\vec{I}}^{B / B^{*}} & 0 \overrightarrow{\vec{U}} \\
0 \overrightarrow{\vec{U}} & m_{B} \overrightarrow{\vec{U}}
\end{array}\right]
\end{aligned}
$$

where the left superscript $N$ is the frame of reference, $B$ is the body name with $B^{*}$ denoting the center of mass, ${ }^{B} \vec{I}^{B / B^{*}}$ is the central inertia dyadic, $m_{B}$ is the body's mass, and $\overrightarrow{\vec{U}}$ is a unity dyadic.

Thus, a spatial momentum could be defined as the dot product of spatial inertia with spatial velocity

$$
\mathcal{I}^{B} \cdot{ }^{N} \mathcal{V}^{B}=\left[\begin{array}{c}
{ }^{B} \overrightarrow{\vec{I}}^{B / B^{*}} \cdot{ }^{N} \overrightarrow{\boldsymbol{\omega}}^{B} \\
m_{B} \cdot{ }^{N} \vec{v}^{B^{*}}
\end{array}\right]
$$

not to be confused with the tensor multiplication $\mathcal{I}^{B N} \mathcal{V}^{B}$, which would instead produce two third-order tensors.

Note that the transpose of a spatial quantity implies an interchange of basis vectors (an interchange of indices on dyadic quantities).

\subsection{Body Sets}

A general multibody tree system, such as that shown in Figure 1, allows a number of useful body sets to be defined. We note that each body $k$ in the system:

- has a single parent body $p[k]$, except the base body, which has the inertial reference $N$ as its parent

- has a set of child bodies $c h[k]$, which may be empty

- is said to be a terminal body if $c h[k]$ is empty

- has an ancestral body set $A n[k]$, which contains the body's parent $p[k]$, that body's parent $p[p[k]]$, and subsequent parent bodies back to and including the base body 


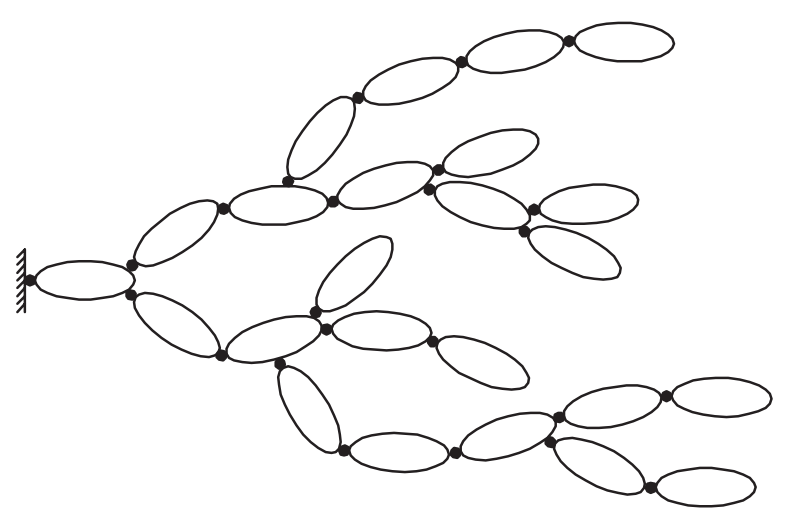

FIGURE 1. System schematic of an arbitrary tree system.

- has a descendant body set denoted Des[ $k]$, which contains the body's children $c h[k]$, the children's children $c b[c b[k]]$, and subsequent child bodies out to and including terminal bodies

\subsection{Recursive Kinematics}

Many recursive definitions in the kinematics of multibody systems are trivial, occurring naturally through the use of relative coordinates. However, before these relationships are expressed in the spatial notation of Section 1, the velocities and accelerations are decomposed into portions, which are explicit in the coordinate velocities, their derivatives, and all remaining terms. Furthermore, observing linear transformations from coordinate velocities $\dot{q}$ and accelerations $\ddot{q}$ to generalized speeds $u$ and their derivatives $\dot{u}$, (as is commonly done with Kane's Method [24]) allows increased efficiency [25] without loss of generality.

The velocity and acceleration decompositions are given by

$$
\begin{aligned}
& { }^{F} \mathcal{V}^{k}={ }^{F} \widehat{\mathcal{V}}^{k}+{ }^{F} \mathcal{V}_{t}^{k} \\
& { }^{F} \mathcal{A}^{k}={ }^{F} \widehat{\mathcal{A}}^{k}+{ }^{F} \mathcal{A}_{t}{ }^{k},
\end{aligned}
$$

where ${ }^{F} \mathcal{V}_{t}^{k}$ and ${ }^{F} \mathcal{A}_{t}^{k}$ are the portions of the velocity and acceleration that are not explicit in the generalized speeds $u$ and their derivatives $\dot{u}$, respectively. The ${ }^{F} \hat{\mathcal{V}}^{k}$ and ${ }^{F} \hat{\mathcal{A}}^{k}$ have the following recursive definitions

$$
\begin{aligned}
& { }^{F} \widehat{\mathcal{V}}^{k}=\left(\mathcal{S}^{k}\right)^{T} \cdot{ }^{F} \hat{\mathcal{V}}^{p[k]}+\mathcal{P}_{k}^{k} u_{k} \\
& { }^{F} \widehat{\mathcal{A}}^{k}=\left(\mathcal{S}^{k}\right)^{T} \cdot{ }^{F} \widehat{\mathcal{A}}^{p[k]}+\mathcal{P}_{k}^{k} \dot{u}_{k}
\end{aligned}
$$

where $u_{k}$ is the coordinate generalized speed, $\mathcal{P}_{k}^{k}$ is the spatial partial velocity vector, and $\mathcal{S}^{k}$ is the spatial shift $d y$ adic, which represents the position vector cross-product coupling of linear and angular relationships.

An arbitrary spatial partial velocity $\mathcal{P}_{r}^{k}$ can be constructed as:

$$
\mathcal{P}_{r}^{k}=\frac{\partial\left({ }^{N} \mathcal{V}^{k}\right)}{\partial u_{r}}= \begin{cases}\left({ }^{r} \mathcal{S}^{k}\right)^{T} \cdot \mathcal{P}_{r}^{r} & r \in k \cup A n[k] \\ 0 & \text { else }\end{cases}
$$

where

$$
{ }^{r} \mathcal{S}^{k}= \begin{cases}\mathcal{U} & r=k \\ \mathcal{S}^{c b[r]} \ldots \mathcal{S}^{p[p[\ldots p[p[k]] \cdots]]} \ldots \mathcal{S}^{p[k]} \cdot \mathcal{S}^{k} & r \in A n[k] \\ \varnothing & \text { else }\end{cases}
$$

And $\mathcal{U}$ is the spatial unity

$$
\mathcal{U}=\left[\begin{array}{cc}
\overrightarrow{\vec{U}} & 0 \\
0 & \overrightarrow{\vec{U}}
\end{array}\right]
$$

We note that the quantities ${ }^{N} \hat{\mathcal{A}}^{k},{ }^{N} \mathcal{A}_{t}^{k}, \mathcal{P}_{k}^{k}$, and $\mathcal{S}^{k}$ for all bodies $k$ can be formed in $O(n)$ operations for a given system state.

\subsection{Recursive Dynamics}

The spatial form of the equations of motion for a multibody tree system containing only single degree of freedom joints is given by Kane's Method [24] as 


$$
\sum_{k=1}^{n}\left(\mathcal{P}_{r}^{k}\right)^{T} \cdot\left(\mathcal{I}^{k} \cdot{ }^{N} \widehat{\mathcal{A}}^{k}-\mathcal{F}^{k}\right)=0
$$

where $\mathcal{F}^{k}$ contains all forces and moments on body $k$ applied about the center of mass as well as the inertial force contributions known in terms of the system state.

The equation of motion (7) readily reduces to (8) by observing the partial velocity decomposition given in (5).

$$
\left(\mathcal{P}_{r}^{r}\right)^{T} \cdot \sum_{k \in D e s[r] \cup r}{ }^{r} \mathcal{S}^{k} \cdot\left(\mathcal{I}^{k} \cdot N \widehat{\mathcal{A}}^{k}-\mathcal{F}^{k}\right)=0
$$

One may now make the assumption that the form of the $k^{\text {th }}$ equation of motion can be expressed as

$$
\left(\mathcal{P}_{k}^{k}\right)^{T} \cdot\left(\widehat{\mathcal{I}}^{k} \cdot{ }^{N} \widehat{\mathcal{A}}^{k}-\widehat{\mathcal{F}}^{k}\right)=0
$$

Thus, trivial boundary data is extracted from the equations of motion associated with terminal bodies $t$

$$
\widehat{\mathcal{I}}^{t}=\mathcal{I}^{t} \quad \text { and } \quad \widehat{\mathcal{F}}^{t}=\mathcal{F}^{t}
$$

Substituting the recursive form for ${ }^{N} \hat{\mathcal{A}}^{k}$ of (4) into (10) gives

$$
\left(\mathcal{P}_{k}^{k}\right)^{T} \cdot\left[\widehat{\mathcal{I}}^{k} \cdot\left(\left(\mathcal{S}^{k}\right)^{T} \cdot{ }^{N} \widehat{\mathcal{A}}^{p[k]}+\mathcal{P}_{k}^{k} \dot{u}_{k}\right)-\widehat{\mathcal{F}}^{t}\right]=0
$$

And solving for $\dot{u}_{k}$ yields

$$
\dot{u}_{k}=-\left(\widehat{\mathcal{M}}^{k}\right)^{-1}\left(\mathcal{P}_{k}^{k}\right)^{T} \cdot\left(\hat{\mathcal{I}}^{k} \cdot\left(\mathcal{S}^{k}\right)^{T} \cdot{ }^{N} \widehat{\mathcal{A}}^{p[k]}-\widehat{\mathcal{F}}^{k}\right)
$$

with

$$
\widehat{\mathcal{M}}^{k}=\left(\mathcal{P}_{k}^{k}\right)^{T} \cdot \widehat{\mathcal{I}}^{k} \cdot \mathcal{P}_{k}^{k}
$$

Now performing similar operations on the equation of motion associated with the parent body's degree of freedom, and substituting the previous solution (12) follows as

$$
\begin{aligned}
0= & \left(\mathcal{P}_{p[k]}^{p[k]}\right)^{T} \cdot\left[\sum_{j \in D e s[p[k]] \cup p[k]}{ }^{p[k]} \mathcal{S}^{j} \cdot\left(\mathcal{I}^{j} \cdot{ }^{N} \hat{\mathcal{A}}^{j}-\mathcal{F}^{j}\right)\right] \\
= & \left(P_{p[k]}^{p[k]}\right)^{T} \cdot\left[\left(I^{p[k]} \cdot{ }^{N} \widehat{\mathcal{A}}^{p[k]}-F^{p[k]}\right)\right. \\
& \left.+\sum_{j \in D e s[p[k]]} p^{[k]} \mathcal{S}^{j} \cdot\left(\mathcal{I}^{j} \cdot{ }^{N} \widehat{\mathcal{A}}^{j}-\mathcal{F}^{j}\right)\right] \\
= & \left(\mathcal{P}_{p[k]}^{p[k]}\right)^{T} \cdot\left[\left(\mathcal{I}^{p[k]} \cdot{ }^{N} \widehat{\mathcal{A}}^{p[k]}-\mathcal{F}^{p[k]}\right)\right. \\
& \left.+\sum_{j \in c b[p[k]]} \mathcal{S}^{j} \cdot\left(\widehat{\mathcal{I}}^{j} \cdot{ }^{N} \widehat{\mathcal{A}}^{j}-\widehat{\mathcal{F}}^{j}\right)\right] \\
= & \left(\mathcal{P}_{p[k]}^{p[k]}\right)^{T} \cdot\left[\left(\mathcal{I}^{p[k]} \cdot{ }^{N} \widehat{\mathcal{A}}^{p[k]}-\mathcal{F}^{p[k]}\right)\right. \\
& \left.+\sum_{j \in c b[p[k]]} \mathcal{S}^{j} \cdot\left(\hat{\mathcal{I}}^{j} \cdot\left\{\left(\mathcal{S}^{j}\right)^{T} \cdot{ }^{N} \widehat{\mathcal{A}}^{p[k]}+\mathcal{P}_{j}^{j} \dot{u}_{j}\right\}-\widehat{\mathcal{F}}^{j}\right)\right] \\
= & \left(\mathcal{P}_{p[k]}^{p[k]}\right)^{T} \cdot\left[\left(\mathcal{I}^{p[k]} \cdot{ }^{N} \widehat{\mathcal{A}}^{p[k]}-\mathcal{F}^{p[k]}\right)\right. \\
& +\sum_{j \in c b[p[k]]} \mathcal{S}^{j} \cdot\left(\widehat { \mathcal { I } } ^ { j } \cdot \left\{\left(\mathcal{S}^{j}\right)^{T} \cdot{ }^{N} \widehat{\mathcal{A}}^{p[k]}-\mathcal{P}_{j}^{j}\left(\widehat{\mathcal{M}}^{j}\right)^{-1}\left(\mathcal{P}_{j}^{j}\right)^{T}\right.\right. \\
& \left.\left.\left.+\left(\widehat{\mathcal{I}}^{j} \cdot\left(\mathcal{S}^{j}\right)^{T} \cdot{ }^{N} \widehat{\mathcal{A}}^{p[k]}-\widehat{\mathcal{F}}^{j}\right)\right\}-\widehat{\mathcal{F}}^{j}\right)\right] \\
= & \left(\mathcal{P}_{p[k]}^{p[k]}\right)^{T} \cdot\left(\widehat{\mathcal{I}}^{p[k]} \cdot{ }^{N} \widehat{\mathcal{A}}^{p[k]}-\widehat{\mathcal{F}}^{p[k]}\right)
\end{aligned}
$$

and the recursive intermediate quantities are obtained through grouping of terms as

$$
\begin{aligned}
& \widehat{\mathcal{I}}^{p[k]}=\mathcal{I}^{p[k]}+\sum_{j \in c b[p[k]]} \mathcal{T}^{j} \cdot \widehat{\mathcal{I}}^{j} \cdot\left(\mathcal{S}^{j}\right)^{T} \\
& \widehat{\mathcal{F}}^{p[k]}=\mathcal{F}^{p[k]}+\sum_{j \in c b[p[k]]} \mathcal{T}^{j} \cdot \widehat{\mathcal{F}}^{j} \\
& \mathcal{T}^{j}=\mathcal{S}^{j} \cdot\left[\mathcal{U}-\widehat{\mathcal{I}}^{j} \cdot \mathcal{P}_{k}^{k}\left(\widehat{\mathcal{M}}^{j}\right)^{-1}\left(\mathcal{P}_{j}^{j}\right)^{T}\right]
\end{aligned}
$$

The $O(n)$ solution then involves calculating the recursive intermediate quantities (20) and (21) inward from the terminal bodies, effectively lower triangularizing 
the system mass matrix. Then forward substituting for the solution using equations (12) and (4) noting that ${ }^{N} \hat{\mathcal{A}}^{N}=0$.

\section{RECURSIVE COORDINATE REDUCTION}

The traditional " $O(n)$ " constraint technique (which actually performs as $O\left(n+n m^{2}+m^{3}\right)$ where $m$ is the number of independent algebraic constraint relations [23]) cuts joints to produce tree topologies from general multibody configurations containing kinematic loops. By contrast, the method of recursive coordinate reduction generates an associated tree topology through the introduction of phantom bodies, which are massless copies of the loop base body (the first body outboard of the inertial reference that branches to form the loop). This construction is demonstrated in Figure 2 as well as a local loop numbering scheme.

\subsection{Kinematics}

"Open loop" systems resulting from the addition of a phantom body to a previously "closed loop" are subject to the velocity and acceleration constraints

$0_{i} \mathcal{V}^{n_{i}}=0$

$$
{ }^{0} \mathcal{A}^{n i}=0
$$

The velocity constraint may be decomposed exactly as with the unconstrained system, resulting in

$$
\begin{aligned}
& 0={ }^{0 i} \mathcal{V}^{n_{i}}={ }^{0_{i}} \hat{\mathcal{V}}^{n_{i}}+{ }^{0_{i}} \mathcal{V}_{t}^{n_{i}} \\
& 0=\left(\mathcal{S}^{n_{i}}\right)^{T} \cdot{ }^{0} \hat{\mathcal{V}}^{n_{i}-1}+\mathcal{P}_{n_{i}}^{n_{i}} u_{n_{i}}+{ }^{0_{i}} \mathcal{V}_{t}^{n_{i}}
\end{aligned}
$$

$u_{n i}$ is always a valid choice for a dependent coordinate, and therefore it can be solved for as

$$
u_{n_{i}}=-\mathcal{Q}^{n_{i}}\left(\mathcal{P}_{n_{i}}^{n_{i}}\right)^{T} \cdot\left[\left(\mathcal{S}^{n_{i}}\right)^{T} \cdot{ }^{0} \hat{\mathcal{V}}^{n_{i}-1}+{ }^{0_{i}} \mathcal{V}_{t}^{n_{i}}\right]
$$

with

$$
\mathcal{Q}^{n_{i}}=\left[\left(\mathcal{P}_{n_{i}}^{n_{i}}\right)^{T} \cdot \mathcal{P}_{n_{i}}^{n_{i}}\right]^{-1}
$$

Equation (27) can be substituted back into (26) to obtain the $n_{i}-1^{\text {st }}$ form of the velocity constraint

$$
0=\mathcal{R}^{n_{i}} \cdot\left[\left({ }^{n_{i}-1} \mathcal{S}^{n_{i}}\right)^{T} \cdot{ }^{0} \hat{\mathcal{V}}^{n_{i}-1}+{ }^{0_{i}} \mathcal{V}_{t}^{n_{i}}\right]
$$
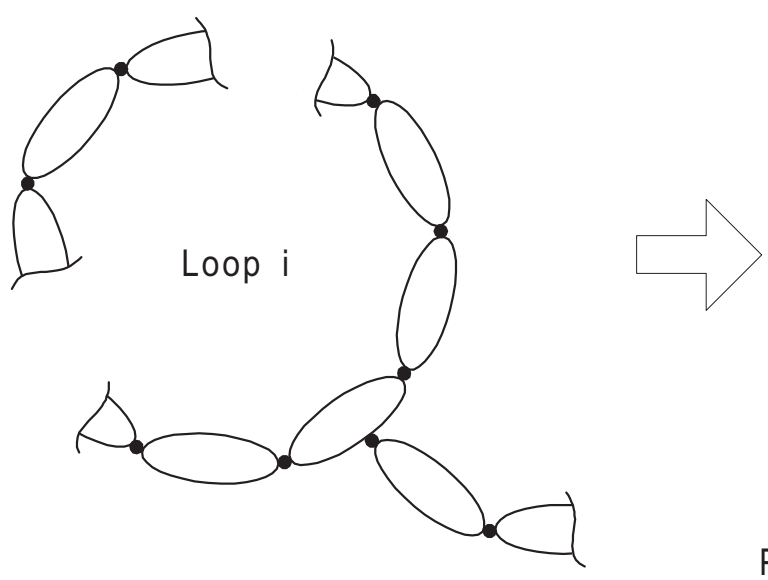

FIGURE 2. Opening a closed loop using a phantom body 
where

$$
\mathcal{R}^{n_{i}}=\mathcal{U}-\mathcal{P}_{n_{i}}^{n_{i}} \mathcal{Q}^{n_{i}}\left(\mathcal{P}_{n_{i}}^{n_{i}}\right)^{T}
$$

Making an appropriate selection for the recursive form of the $k_{i}^{\text {th }}$ equation and solving follows as

$$
\begin{aligned}
0= & \mathcal{R}^{k_{i}+1} \cdot\left[\left({ }^{k_{i}} \mathcal{S}^{n_{i}}\right)^{T} \cdot{ }^{0} \hat{\mathcal{V}}^{k_{i}}+{ }^{0_{i}} \mathcal{V}_{t}^{n_{i}}\right] \\
= & \mathcal{R}^{k_{i}+1} \cdot\left[\left({ }^{k_{i}} \mathcal{S}^{n_{i}}\right)^{T} \cdot \mathcal{P}_{k_{i}}^{k_{i}} u_{k_{i}}+\left({ }^{k_{i}-1} \mathcal{S}^{n_{i}}\right)^{T}\right. \\
& \left.\cdot{ }^{0_{i}} \hat{\mathcal{V}}^{k_{i}-1}+{ }^{0_{i}} \mathcal{V}_{t}^{n_{i}}\right]
\end{aligned}
$$

And therefore the general solution

$$
\begin{array}{r}
u_{k_{i}}=-\mathcal{Q}^{k_{i}}\left(\mathcal{P}_{k_{i}}^{k_{i}}\right)^{T} \cdot{ }_{i}^{k_{i}} \mathcal{S}^{n_{i}} \cdot \mathcal{R}^{k_{i}+1} \\
\cdot\left[\left({ }^{k_{i}-1} \mathcal{S}^{n_{i}}\right)^{T} \cdot{ }^{0_{i}} \widehat{\mathcal{V}}^{k_{i}-1}+{ }^{0_{i}} \mathcal{V}_{t}^{n_{i}}\right]
\end{array}
$$

is obtained with

$$
\mathcal{Q}^{k_{i}}=\left[\left(\mathcal{P}_{k_{i}}^{k_{i}}\right)^{T} \cdot{ }^{k_{i}} \mathcal{S}^{n_{i}} \cdot \mathcal{R}^{k_{i}+1} \cdot\left({ }^{k_{i}} \mathcal{S}^{n_{i}}\right)^{T} \cdot \mathcal{P}_{k_{i}}^{k_{i}}\right]^{-1}
$$

(under the assumption that the last $m_{i}$ consecutive coordinates constitute a valid selection of dependent coordinates-e.g., the first $p_{i}$ coordinates form a valid set of independent coordinates).

Satisfying the $k_{i}-1^{\text {st }}$ equation

$$
0=\mathcal{R}^{k_{i}+1} \cdot\left[\left({ }^{k_{i}} \mathcal{S}^{n_{i}}\right)^{T} \cdot \cdot_{i} \hat{\mathcal{V}}^{k_{i}}+{ }^{0_{i}} \mathcal{V}_{t}^{n_{i}}\right]
$$

reveals the recursive relationship

$$
\begin{aligned}
\mathcal{R}^{k_{i}}= & \mathcal{R}^{k_{i}+1} \cdot\left[\mathcal{U}-\left({ }^{k_{i}} \mathcal{S}^{n_{i}}\right)^{T}\right. \\
& \left.\cdot \mathcal{P}_{k_{i}}^{k_{i}} \mathcal{Q}^{k_{i}}\left(\mathcal{P}_{k_{i}}^{k_{i}}\right)^{T} \cdot{ }^{k_{i}} \mathcal{S}^{n_{i}} \cdot \mathcal{R}^{k_{i}+1}\right]
\end{aligned}
$$

For the dependent bodies, we note that in the usual $\hat{\mathcal{V}}$ decomposition of (37) it is valid to substitute (33), resulting in (38)

$$
{ }^{0_{i}} \hat{\mathcal{V}}^{k_{i}}=\left(\mathcal{S}^{k_{i}}\right)^{T} \cdot{ }^{0} \hat{\mathcal{V}}^{k_{i}-1}+\mathcal{P}_{k_{i}}^{k_{i}} u_{k_{i}}
$$

$$
=\mathcal{W}^{k_{i}} \cdot{ }^{0_{i}} \widehat{\mathcal{V}}^{k_{i}-1}+\mathcal{X}^{k_{i}} \cdot{ }^{0_{i}} \widehat{\mathcal{V}}_{{ }^{\prime}{ }^{n}}
$$

with

$$
\mathcal{W}^{k_{i}}=\left({ }^{k_{i}-1} \mathcal{S}^{k_{i}}\right)^{T}+\mathcal{X}^{k_{i}} \cdot\left({ }^{k_{i}-1} \mathcal{S}^{n_{i}}\right)^{T}
$$

$$
\mathcal{X}^{k_{i}}=-\mathcal{P}_{k_{i}}^{k_{i}} \mathcal{Q}^{k_{i}}\left(\mathcal{P}_{k_{i}}^{k_{i}}\right)^{T} \cdot k_{i} \mathcal{S}^{n_{i}} \cdot \mathcal{R}^{k_{i}+1}
$$

The relationship (38) can then be used to write additional recursive relations expressing the dependent velocities in terms of the last independent coordinate in the loop $p_{i}$

$$
\begin{aligned}
& { }^{0_{i}} \hat{\mathcal{V}}^{k_{i}}=\mathcal{Y}^{k_{i}} \cdot{ }^{0_{i}} \hat{\mathcal{V}}^{P_{i}}+\mathcal{Z}^{k_{i}} \cdot{ }^{0_{i}} \mathcal{V}_{t}^{n_{i}} \\
& \mathcal{Y}^{k_{i}}=\mathcal{W}^{k_{i}} \cdot \mathcal{Y}^{k_{i}-1}
\end{aligned}
$$

$$
\mathcal{Z}^{k_{i}}=\mathcal{X}^{k_{i}}+\mathcal{W}^{k_{i}} \cdot \mathcal{Z}^{k_{i}-1}
$$

with the trivial boundary data

$$
\mathcal{Y}^{p_{i}}=\mathcal{U} \quad \text { and } \quad \mathcal{Z}^{p_{i}}=0
$$

This same procedure can be followed with the dependent accelerations resulting in

$$
{ }^{0} \widehat{\mathcal{A}}^{k_{i}}=\mathcal{Y}^{k_{i}} \cdot{ }^{0_{i}} \widehat{\mathcal{A}}^{p_{i}}+\mathcal{Z}^{k_{i}} \cdot{ }^{0_{i}} \mathcal{A}_{t}^{n_{i}}
$$




\subsection{Dynamics}

The constrained form of the equation of motion is given by

$$
0=\sum_{k=1}^{n}\left(\widetilde{\mathcal{P}}_{r}^{k}\right)^{T} \cdot\left(\mathcal{I}^{k} \cdot{ }^{N} \widehat{\mathcal{A}}^{k}-\mathcal{F}^{k}\right)
$$

where $\tilde{\mathcal{P}}$ are the constrained non-holonomic spatial partial velocities (which explicitly account for the constraint through direct embedding of constraints into the equations of motion).

Here we note the additional kinematic definitions

$$
{ }^{N} \widehat{\mathcal{A}}^{k_{i}}=\left({ }^{0_{i}} \mathcal{S}^{k_{i}}\right)^{T} \cdot{ }^{N} \widehat{\mathcal{A}}^{0_{i}}+{ }^{0_{i}} \widehat{\mathcal{A}}^{k_{i}}
$$

$$
{ }^{N} \widehat{\mathcal{V}}^{k_{i}}=\left({ }^{0_{i}} \mathcal{S}^{k_{i}}\right)^{T} \cdot{ }^{N} \widehat{\mathcal{V}}^{0_{i}}+{ }^{0_{i}} \widehat{\mathcal{V}}^{k_{i}}
$$

Substituting the acceleration relationship (47) into (46) and restricting our attention to the local loop we obtain

$$
\begin{aligned}
0= & \sum_{k_{i}=r}^{n_{i}}\left(\widetilde{\mathcal{P}}_{r^{k_{i}}}\right)^{T} \cdot\left(\mathcal{I}^{k_{i}} \cdot\left({ }^{0_{i}} \mathcal{S}^{k_{i}}\right)^{T}\right. \\
& \left.\cdot{ }^{N} \widehat{\mathcal{A}}^{0_{i}}+\mathcal{I}^{k_{i}} \cdot{ }^{0_{i}} \widehat{\mathcal{A}}^{0_{i}}-\mathcal{F}^{k_{i}}\right)
\end{aligned}
$$

The constrained partial velocities are the partial derivatives of the velocities written in terms of the independent coordinates as in (41). These partial derivatives are

$$
\widetilde{\mathcal{P}}_{r^{k_{i}}}^{k_{i}}=\frac{\partial\left({ }^{N} \mathcal{V}^{k_{i}}\right)}{\partial u_{r}}= \begin{cases}\mathcal{P}_{r}^{k_{i}} & k_{i} \in \text { ind }_{i} \text { and } r \in \text { ind }_{i} \\ \mathcal{Y}^{k_{i}} \cdot \mathcal{P}_{r}^{p_{i}} & k_{i} \in \text { dep }_{i} \text { and } r \in \text { ind }_{i} \\ 0 & r \in \text { dep }_{i}\end{cases}
$$

where $i_{i} d_{i}$ and $d e p_{i}$ are the sets of independent and dependent coordinatees (respectively) on local loop $i$.
Substitution of (50) into (49) results in

$$
\begin{aligned}
& 0=\sum_{k_{i}=r}^{p_{i}-1}\left(\mathcal{P}_{r}^{k_{i}}\right)^{T} \cdot\left(\mathcal{I}^{k_{i}} \cdot\left({ }^{0_{i}} \mathcal{S}^{k_{i}}\right)^{T} \cdot{ }^{N} \widehat{\mathcal{A}}^{0_{i}}+\mathcal{I}^{k_{i}} \cdot{ }^{0_{i}} \widehat{\mathcal{A}}^{{ }_{i}}-\mathcal{F}^{k_{i}}\right) \\
& +\left(\mathcal{P}_{r}^{p_{i}}\right)^{T} \cdot \sum_{k_{i}=p_{i}}^{n_{i}}\left(\mathcal{Y}^{k_{i}}\right)^{T} \cdot\left(\mathcal{I}^{k_{i}} \cdot\left({ }^{0_{i}} \mathcal{S}^{k_{i}}\right)^{T} \cdot{ }^{N} \widehat{\mathcal{A}}^{0_{i}}+\mathcal{I}^{k_{i}} \cdot{ }^{0_{i}} \widehat{\mathcal{A}}^{k}-\mathcal{F}^{k_{i}}\right)
\end{aligned}
$$

And ${ }^{{ }^{0}} \widehat{\mathcal{A}}^{k}{ }^{k}$ for the dependent bodies is given in terms of ${ }^{0_{i}} \widehat{\mathcal{A}}^{p_{i}}$ by equation (45), which allows us to rewrite (51) as

$0=\sum_{k_{i}=r}^{p_{i}}\left(\mathcal{P}_{r}^{k_{i}}\right)^{T} \cdot\left(\breve{\mathcal{I}}^{k_{i} ; 0_{i}} \cdot{ }^{N} \widehat{\mathcal{A}}^{0_{i}}+{ }^{0_{i}} \breve{\mathcal{I}}^{k_{i}} \cdot{ }^{0_{i}} \widehat{\mathcal{A}}^{k_{i}}-\breve{\mathcal{F}}^{k_{i}}\right)$

with

$\breve{\mathcal{I}}^{k_{i}, 0_{i}}= \begin{cases}\mathcal{I}^{k_{i}} \cdot\left({ }^{0_{i}} \mathcal{S}^{k_{i}}\right)^{T} & k_{i} \neq p_{i} \\ \sum_{j_{i}=p_{i}}^{n_{i}}\left(\mathcal{Y}^{j_{i}}\right)^{T} \cdot \mathcal{I}^{j_{i}} \cdot\left({ }^{0_{i}} \mathcal{S}^{j_{i}}\right)^{T} & k_{i}=p_{i}\end{cases}$

${ }^{0_{i}} \breve{\mathcal{I}}^{k_{i}}= \begin{cases}I^{k_{i}} & k_{i} \neq p_{i} \\ \sum_{j_{i}=p_{i}}^{n_{i}}\left(\mathcal{Y}^{j_{i}}\right)^{T} \cdot \mathcal{I}^{j_{i}} \cdot \mathcal{Y}^{j_{i}} & k_{i}=p_{i}\end{cases}$

$\breve{\mathcal{F}}^{k_{i}}=\left\{\begin{array}{lr}\mathcal{F}^{k_{i}} & k_{i} \neq p_{i} \\ \sum_{j_{i}=p_{i}}^{n_{i}}\left(\mathcal{Y}^{j_{i}}\right)^{T} \cdot\left[\mathcal{F}^{j_{i}}-\mathcal{I}^{j_{i}} \cdot \mathcal{Z}^{j_{i}} \cdot{ }^{0_{i}} \mathcal{A}_{t}^{n_{i}}\right] & k_{i}=p_{i}\end{array}\right.$

The coordinate reduced equation of motion (52) may then be solved recursively in exactly the same fashion as the unconstrained equations of Section 4. That is, substitution of (5) into the $j_{i}^{\text {th }}$ equation of motion (52) results in

$$
\begin{aligned}
0 & =\left(\mathcal{P}_{j_{i}}^{j_{i}}\right)^{T} \cdot \sum_{k_{i}=j_{i}}^{p_{i}}\left({ } ^ { j _ { i } } \mathcal { S } ^ { k _ { i } } \cdot \left(\breve{\mathcal{I}}^{k_{i} ; 0_{i}} \cdot{ }^{N} \widehat{\mathcal{A}}^{0_{i}}+{ }^{0_{i}} \breve{\mathcal{I}}^{k_{i}}\right.\right. \\
& \left.\left.\cdot{ }^{0_{i}} \widehat{\mathcal{A}}^{k_{i}}-\mathcal{F}^{k_{i}}\right)\right)
\end{aligned}
$$


And again, the recursive assumption is that the $j_{i}^{\text {th }}$ equation is of the form

$$
0=\left(\mathcal{P}_{j_{i}}^{j_{i}}\right)^{T} \cdot\left(\widehat{\mathcal{I}}^{j_{i} 0_{i}} \cdot{ }^{N} \widehat{\mathcal{A}}^{0_{i}}+{ }^{0_{i}} \widehat{\mathcal{I}}^{j_{i}} \cdot{ }_{i}{ }^{i_{\mathcal{A}}} \widehat{\mathcal{A}}^{j_{i}}-\widehat{F}^{j_{i}}\right)
$$

and trivial boundary data is obtained for the last independent coordinate in the loop $p_{i}$

$$
\widehat{\mathcal{I}}^{p_{i} ; 0_{i}}=\breve{\mathcal{I}}^{p_{i} ; 0_{i}} \quad 0_{i} \widehat{\mathcal{I}}^{p_{i}}={ }^{0} \breve{\mathcal{I}}^{p_{i}} \quad \widehat{\mathcal{F}}^{p_{i}}=\breve{\mathcal{F}}^{p_{i}}
$$

The recursive form of ${ }^{0_{i}} \widehat{\mathcal{A}}^{j_{i}}$ from (4) is then substituted into (57), and the unknown $\dot{u}_{j i}$ is solved for in terms of the dynamics of the parent body.

$$
\begin{aligned}
\dot{u}_{j_{i}}= & -\left(\widehat{\mathcal{M}}^{j_{i}}\right)^{-1}\left(\mathcal{P}_{j_{i}}^{j_{i}}\right)^{T} \cdot\left(\widehat{\mathcal{I}}^{j_{i}} 0_{i} \cdot{ }^{N} \widehat{\mathcal{A}}^{0_{i}}+{ }^{0_{i}} \widehat{\mathcal{I}}^{j_{i}}\right. \\
& \left.\cdot\left(\mathcal{S}^{j_{i}}\right)^{T} \cdot{ }^{0_{i}} \widehat{\mathcal{A}}^{p\left[j_{i}\right]}-\widehat{\mathcal{F}}^{p_{i}}\right)
\end{aligned}
$$

In the equation of motion associated with the parent of the $j_{i}^{\text {th }}$ body (e.g., [56] with $j_{i}$ replaced by $j_{i}-1$ ), the first term can be extracted from the sum and the remaining sum replaced through appropriate substitution of the recursive quantities in (57). Further substituting the solution of $\dot{u}_{j i}$ and grouping terms reveals the solution for the recursive relationships

$$
\begin{aligned}
& \widehat{\mathcal{I}}^{j_{i}-1 ; 0_{i}}=\breve{\mathcal{I}}^{j_{i}-1 ; 0_{i}}+\mathcal{T}^{j_{i}} \cdot \widehat{\mathcal{I}}^{j_{i} ; 0_{i}} \\
& { }^{0_{i}} \widehat{\mathcal{I}}^{j_{i}-1}={ }^{0_{i}} \widehat{\mathcal{I}}^{j_{i}-1}+\mathcal{T}^{j_{i}} \cdot{ }^{0_{i}} \widehat{\mathcal{I}}^{j_{i}} \cdot\left(\mathcal{S}^{j_{i}}\right)^{T} \\
& \widehat{\mathcal{F}}^{j_{i}-1}=\mathcal{F}^{j_{i}-1}+\mathcal{T}^{j_{i}} \cdot \widehat{\mathcal{F}}^{j_{i}} \\
& \mathcal{T}^{j_{i}}=\mathcal{S}^{j_{i}} \cdot\left[\mathcal{U}-{ }^{0_{i}} \widehat{\mathcal{I}}^{j_{i}} \cdot \mathcal{P}_{j_{i}}^{j_{i}}\left(\widehat{\mathcal{M}}^{j_{i}}\right)^{-1}\left(\mathcal{P}_{j_{i}}^{j_{i}}\right)^{T}\right] \\
& \widehat{\mathcal{M}}^{j_{i}}=\left(\mathcal{P}_{j_{i}}^{j_{i}}\right)^{T} \cdot \cdot_{i} \widehat{\mathcal{I}}^{j_{i}} \cdot \mathcal{P}_{j_{i}}^{j_{i}}
\end{aligned}
$$

At the loop base body we note that ${ }^{0_{i}} \widehat{\mathcal{A}}^{0_{i}}=0$, thereby dropping the additional term and following the definitions for unconstrained tree systems (20-22). An additional recursive triangularization of equations (not shown) is also required and trivially obtained when observing that the partial derivatives of (50) change form on the loop base body.

The most constraining limitation of this, the RCR formulation, is the assumption that the last $m_{i}$ coordinates of an opened loop constitute a valid selection of dependent coordinates. For this reason, solutions for many looped multibody systems cannot be modeled by the presented form of the RCR, and many more have the possibility of entering singular configurations and/or areas of numerical ill conditioning during simulation. Two methods for avoiding singularities by altering the dependent coordinate selection within the framework of the RCR will be described and evaluated in the following sections.

\section{ARBITRARY SELECTION OF DEPENDENT COORDINATES}

The first method of avoiding singularities associated with the RCR involves the specification of arbitrary dependent coordinates directly in the formulation of the RCR algorithm. In other words, independent generalized coordinates will be included between the dependent coordinates. We also note that the last coordinate in the loop can and will always be chosen as dependent. This is because not doing so would require the phantom body to have mass properties, which, although trivial, adds needless additional complexity to the formulation (e.g., some steps can be skipped by noting that the spatial inertia associated with body $n_{i}$ is always zero). Figure 3 illustrates a possible selection of dependent coordinates as well as additional numbering information that will be required by the new formulation.

The kinematic solution (36), which solves for the behavior of a coordinate in a loop, involves the inversion of an independent constraint relationship. Because there are exactly $m_{i}$ independent constraint equations associated with loop $i$, this inversion is not admissible for the independent coordinates $f_{i}$ through $l_{i}$ (where $f_{i}$ and $l_{i}$ are the joint coordinates associated with bodies $F_{i}$ and $L_{i}$ of Fig. 3). Instead, contributions from these coordinates appear directly in the kinematics formulation as 
FIGURE 3. Body numbering in the selection of arbitrary dependent coordinates.

$$
\begin{aligned}
0= & \mathcal{R}^{k_{i}+1} \cdot\left[\left({ }^{k_{i}} \mathcal{S}^{n_{i}}\right)^{T} \cdot{ }^{0_{i}} \hat{\mathcal{V}}^{k_{i}}+{ }^{{ }}{ } \mathcal{V}_{t}^{n_{i}}\right. \\
& \left.+\sum_{j \in i \operatorname{Des}\left[k_{i}\right]}\left({ }^{j} \mathcal{S}^{n_{i}}\right)^{T} \cdot \mathcal{P}_{j}^{j} \dot{u}_{j}\right]
\end{aligned}
$$

where $i D e s$ is the set of independent descendants on the local loop (e.g., $i \operatorname{Des}\left[k_{i}\right]=\operatorname{Des}\left[k_{i}\right] \cap i n d_{i}$ ) and

$$
\mathcal{R}^{k_{i}}= \begin{cases}\mathcal{R}^{k_{i}+1} \cdot\left[\begin{array}{ll}
\mathcal{U}-\left({ }^{k_{i}} \mathcal{S}^{n_{i}}\right)^{T} \cdot \mathcal{P}_{k_{i}}^{k_{i}} \mathcal{Q}^{k_{i}}\left(\mathcal{P}_{k_{i}}^{k_{i}}\right)^{T} \\
\operatorname{ki}_{i} \mathcal{S}^{n_{i}} \cdot \mathcal{R}^{k_{i}+1}
\end{array}\right] & k_{i} \in \text { dep }_{i} \\
\mathcal{R}^{k_{i}+1} & k_{i} \in \text { ind }_{i}\end{cases}
$$

Hence, the solution for the dependent generalized accelerations can be obtained by the acceleration form of (65) and are given by

$$
\begin{aligned}
& \dot{u}_{D_{i}}=-\mathcal{Q}^{D_{i}}\left(\mathcal{P}_{D_{i}}^{D_{i}}\right)^{T} \cdot{ }^{D_{i}} \mathcal{S}^{n_{i}} \cdot \mathcal{R}^{D_{i}+1} \\
& \cdot\left[\left({ }^{D_{i}-1} \mathcal{S}^{n_{i}}\right)^{T} \cdot{ }^{0_{i}} \widehat{\mathcal{A}}^{D_{i}}+{ }^{0_{i}} \mathcal{A}_{t}^{n_{i}}+\sum_{j \in i \text { es }\left[D_{i}\right]}\left({ }^{j} \mathcal{S}^{n_{i}}\right)^{T} \cdot \mathcal{P}_{j}^{j} \dot{u}_{j}\right](67)
\end{aligned}
$$

with $\mathcal{W}^{I_{i}}=\left(\mathcal{S}^{I_{i}}\right)^{T}$ and $\mathcal{X}^{I_{i}}=0$.
We now note that the velocities of the bodies associated with the dependent coordinates $D_{i}$ are given by$$
=\mathcal{W}^{I_{i}} \cdot{ }^{0}{ }_{i} \hat{\mathcal{V}}^{I_{i}-1}+\mathcal{X}^{I_{i}} \cdot{ }^{0_{i}} \mathcal{V}_{t}^{n_{i}}+\mathcal{P}_{I_{i}}^{I_{i}} u_{I_{i}}
$$

Combining these two relationships for a general body $k_{i}$ on the loop and outboard of $p_{i}$ (e.g., $\left.k_{i} \in \operatorname{Des}\left[p_{i}\right]\right)$ results in

$$
{ }^{0_{i}} \hat{\mathcal{V}}^{k_{i}}=\mathcal{W}^{k_{i}} \cdot{ }^{0_{i}} \hat{\mathcal{V}}^{k_{i}-1}+\mathcal{X}^{k_{i}} \cdot{ }^{0_{i}} \mathcal{V}_{t}^{n_{i}}+\mathcal{G}^{k_{i}} \cdot \mathcal{K}_{i} \mathbf{u}_{\mathbf{i}}
$$

where $\mathcal{W}^{D_{i}}$ and $\mathcal{X}^{D_{i}}$ are given exactly as in (39-40). And the independent coordinates $I_{i}$ can be cast in a similar form,

$$
{ }^{0_{i}} \hat{\mathcal{V}}^{I_{i}}=\left(\mathcal{S}^{I_{i}}\right)^{T} \cdot{ }^{0_{i}} \hat{\mathcal{V}}^{I_{i}-1}+\mathcal{P}_{I_{i}}^{I_{i}} u_{I_{i}}
$$


where

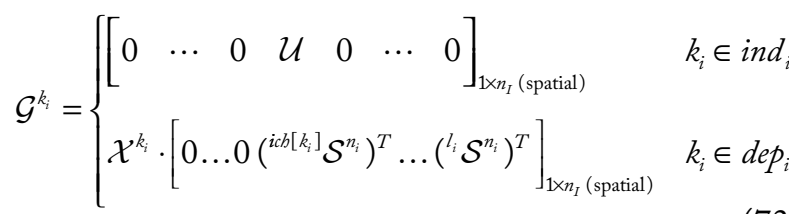

$$
\begin{aligned}
& \mathcal{K}_{i}=\operatorname{diag}\left[\left(\mathcal{P}_{f_{i}}^{f_{i}}\right) \ldots\left(\mathcal{P}_{l_{i}}^{l_{i}}\right)\right]_{n_{I} \times n_{I} \text { (spatial) }} \\
& \mathbf{u}_{\mathrm{i}}=\left[\begin{array}{lll}
u_{f_{i}} & \cdots & u_{l_{i}}
\end{array}\right]_{\left.n_{I} \times 1 \text { (scalar }\right)}^{T} \\
& \mathcal{W}^{k_{i}}= \begin{cases}\left(\mathcal{S}^{k_{i}}\right)^{T} & k_{i} \in \text { ind }_{i} \\
\left(\mathcal{S}^{k_{i}}\right)^{T}+\mathcal{X}^{k_{i}} \cdot\left({ }^{k_{i}-1} \mathcal{S}^{n_{i}}\right)^{T} & k_{i} \in \operatorname{dep}_{i}\end{cases} \\
& \mathcal{X}^{k_{i}}= \begin{cases}0 & k_{i} \in \text { ind }_{i} \\
-\mathcal{P}_{k_{i}}^{k_{i}} \mathcal{Q}^{k_{i}}\left(\mathcal{P}_{k_{i}}^{k_{i}}\right)^{T} \cdot{ }^{k_{i}} \mathcal{S}^{n_{i}} \cdot \mathcal{R}^{k_{i}+1} & k_{i} \in \operatorname{dep}_{i}\end{cases}
\end{aligned}
$$

with $i c h\left[k_{i}\right]=\operatorname{ch}\left[k_{i}\right] \cap i n d_{i}$ and $n_{I}$ equal to the number of independent coordinates outboard of $p_{i}$ (e.g., the number of independents in $f_{i}$ through $l_{i}$ ).

Once again the velocities may be written in terms of $p_{i}$ and the known portion of $n_{i}$, but with an additional contribution from all of the independent variables lying between them $\left(\mathbf{u}_{\mathbf{i}}\right)$.

$$
{ }^{0_{i}} \hat{\mathcal{V}}^{k_{i}}=\mathcal{Y}^{k_{i}} \cdot{ }^{0_{i}} \hat{\mathcal{V}}^{p_{i}}+\mathcal{Z}^{k_{i}} \cdot{ }^{0_{i}} \mathcal{V}_{t}^{n_{i}}+\mathcal{H}^{k_{i}} \cdot \mathcal{K}_{i} \mathbf{u}_{\mathbf{i}}
$$

where

$$
\begin{aligned}
& \mathcal{Y}^{k_{i}}=\mathcal{W}^{k_{i}} \cdot \mathcal{Y}^{k_{i}-1} \\
& \mathcal{Z}^{k_{i}}=\mathcal{X}^{k_{i}}+\mathcal{W}^{k_{i}} \cdot \mathcal{Z}^{k_{i}-1} \\
& \mathcal{H}^{k_{i}}=\mathcal{G}^{k_{i}}+\mathcal{W}^{k_{i}} \cdot \mathcal{H}^{k_{i}-1}
\end{aligned}
$$

(80) with
The constrained equations of motion associated with the last independent coordinates $f_{i}$ through $l_{i}$ of the local loop are given by

$$
0=\sum_{k=p_{i}+1}^{n_{i}}\left(\widetilde{\mathcal{P}}_{r}^{k}\right)^{T} \cdot\left(\mathcal{I}^{k} \cdot{ }^{N} \widehat{\mathcal{A}}^{k}-\mathcal{F}^{k}\right)
$$

or

$$
\begin{aligned}
0 & =\sum_{k=p_{i}+1}^{n_{i}}\left(\widetilde{\mathcal{P}}_{r}^{k}\right)^{T} \cdot\left(\mathcal{I}^{k} \cdot\left({ }^{0_{i}} \mathcal{S}^{k}\right)^{T}\right. \\
& \left.\cdot{ }^{N} \widehat{\mathcal{A}}^{0_{i}}+\mathcal{I}^{k} \cdot{ }^{0_{i}} \widehat{\mathcal{A}}^{k}-\mathcal{F}^{k}\right)
\end{aligned}
$$

It should be clear that

$$
\widetilde{\mathcal{P}}_{r}^{k_{i}}=\mathcal{H}_{r}^{k} \cdot \mathcal{P}_{r}^{r}
$$

where $\mathcal{H}_{r}^{k}$ is the element of $\mathcal{H}^{k}$ corresponding to the contribution of the $r^{\text {th }}$ generalized speed. Using this relationship, Eq. (83) becomes

$$
\begin{aligned}
0= & \left(\mathcal{P}_{r}^{r}\right)^{T} \cdot \sum_{k=p_{i}+1}^{n_{i}}\left(\mathcal{H}_{r}^{k}\right)^{T} \cdot\left(\mathcal{I}^{k} \cdot\left({ }^{0_{i}} \mathcal{S}^{k}\right)^{T}\right. \\
& { }^{N} \widehat{\mathcal{A}}^{0_{i}}+\mathcal{I}^{k} \cdot \mathcal{Y}^{k} \cdot{ }^{0_{i}} \widehat{\mathcal{A}}^{p_{i}}+\mathcal{I}^{k} \cdot \mathcal{Z}^{k} \\
& \left.{ }^{0_{i}} \mathcal{A}_{t}{ }^{n_{i}}+\mathcal{I}^{k} \cdot \mathcal{H}^{k} \cdot \mathcal{K}_{i} \mathbf{u}_{\mathrm{i}}-\mathcal{F}^{k}\right)
\end{aligned}
$$

and grouping terms results in

$$
\begin{aligned}
0 & =\left(\mathcal{P}_{r}^{r}\right)^{T} \cdot \sum_{k=p_{i}+1}^{n_{i}}\left(\mathcal{H}_{r}^{k}\right)^{T} \cdot\left(\breve{\mathcal{I}}^{k_{i} ; 0_{i}} \cdot{ }^{N} \widehat{\mathcal{A}}^{0_{i}}\right. \\
& \left.+{ }^{0_{i}} \breve{\mathcal{I}}^{k} \cdot{ }^{0_{i}} \widehat{\mathcal{A}}^{p_{i}}+\breve{\mathcal{H}}^{k} \cdot \mathcal{K}_{i} \mathbf{u}_{\mathrm{i}}-\breve{\mathcal{F}}^{k}\right)
\end{aligned}
$$

$$
\breve{\mathcal{I}}^{k_{i} ; 0_{i}}=\mathcal{I}^{k} \cdot\left({ }^{0_{i}} \mathcal{S}^{k}\right)^{T}
$$


${ }^{0_{i}} \breve{\mathcal{I}}^{k}=\mathcal{I}^{k} \cdot \mathcal{Y}^{k}$

$\breve{\mathcal{F}}^{k}=\mathcal{F}^{k}-\mathcal{I}^{k} \cdot \mathcal{Z}^{k} \cdot{ }^{0_{i}} \mathcal{A}_{t}^{n_{i}}$

$\breve{\mathcal{H}}^{k}=\mathcal{I}^{k} \cdot \mathcal{H}^{k}$

which can also be written as

$$
\begin{aligned}
0 & =\left(\mathcal{P}_{k_{i}}^{k_{i}}\right)^{T} \cdot\left(\widehat{\mathcal{I}}^{k_{i} ; 0_{i}} \cdot{ }^{N} \widehat{\mathcal{A}}^{0_{i}}+{ }^{0_{i}} \widehat{\mathcal{I}}^{k_{i}} \cdot{ }^{0_{i}} \widehat{\mathcal{A}}^{p_{i}}\right. \\
& \left.+\widehat{\mathcal{H}}^{k_{i}} \cdot \mathcal{K}_{i} \dot{\mathbf{u}}_{\mathrm{i}}-\widehat{\mathcal{F}}^{k_{i}}\right)
\end{aligned}
$$

where for independent coordinates $f_{i}$ through $l_{i}$

$$
\begin{aligned}
& \widehat{\mathcal{I}}^{0_{i} ; k_{i}}=\sum_{j=p_{i}+1}^{n_{i}}\left(\mathcal{H}_{k_{i}}^{j}\right)^{T} \cdot \breve{\mathcal{I}}^{j ; 0_{i}} \\
& 0_{i} \widehat{\mathcal{I}}^{k_{i}}=\sum_{j=p_{i}+1}^{n_{i}}\left(\mathcal{H}_{k_{i}}^{j}\right)^{T} \cdot{ }^{0} \breve{\mathcal{I}}^{j} \\
& \widehat{\mathcal{F}}^{k_{i}}=\sum_{j=p_{i}+1}^{n_{i}}\left(\mathcal{H}_{k_{i}}^{j}\right)^{T} \cdot \breve{\mathcal{F}}^{j} \\
& \widehat{\mathcal{H}}^{k_{i}}=\sum_{j=p_{i}+1}^{n_{i}}\left(\mathcal{H}_{k_{i}}^{j}\right)^{T} \cdot \breve{\mathcal{H}}^{j}
\end{aligned}
$$

And the solutions for the independent generalized accelerations must satisfy the set of linear scalar equations

$$
\begin{aligned}
\widehat{\mathcal{M}}^{k_{i}} \mathbf{u}_{\mathrm{i}}= & -\left(\mathcal{P}_{k_{i}}^{k_{i}}\right)^{T} \cdot\left(\widehat{\mathcal{I}}^{0_{i} ; k_{i}} \cdot{ }^{N} \widehat{\mathcal{A}}^{0_{i}}+{ }^{0_{i}} \widehat{\mathcal{I}}^{k_{i}}\right. \\
& \left.\cdot{ }^{0_{i}} \widehat{\mathcal{A}}^{p_{i}}-\widehat{\mathcal{F}}^{k_{i}}\right)
\end{aligned}
$$

with
$\widehat{\mathcal{M}}_{M} \mathbf{u}_{\mathrm{i}}=-\mathcal{K}_{i}^{T} \cdot\left(\widehat{\mathcal{I}}_{M}^{0_{i}} \cdot{ }^{N} \widehat{\mathcal{A}}^{0_{i}}+\widehat{\mathcal{I}}_{M}^{p_{i}} \cdot{ }^{0_{i}} \widehat{\mathcal{A}}^{p_{i}}-\widehat{\mathcal{F}}_{M}\right)$

where the $\hat{\mathcal{I}}_{M}^{0_{i}}, \widehat{\mathcal{I}}_{M}^{p_{i}}$, and $\widehat{\mathcal{F}}_{M}$ are appropriate column matrices with elements $\widehat{\mathcal{I}}^{0_{i} ; k_{i}},{ }^{0_{i}} \widehat{\mathcal{I}}^{k_{i}}$, and $\widehat{\mathcal{F}}^{k_{i}}$, respectively, and $\widehat{\mathcal{M}}_{M}$ is the square matrix constructed from the $\widehat{\mathcal{M}}^{k_{i}}$ for $k_{i}$ from $f_{i}$ to $l_{i}$.

The solution

$$
\begin{aligned}
\mathbf{u}_{\mathrm{i}}= & -\left(\widehat{\mathcal{M}}_{M}\right)^{-1} \mathcal{K}_{i}^{T} \cdot\left(\widehat{\mathcal{I}}_{M}^{0_{i}} \cdot{ }^{N} \widehat{\mathcal{A}}^{0_{i}}+\widehat{\mathcal{I}}_{M}^{p_{i}}\right. \\
& \left.\cdot{ }^{{ }_{i}} \widehat{\mathcal{A}}^{p_{i}}-\widehat{\mathcal{F}}_{M}\right)
\end{aligned}
$$

$$
\widehat{\mathcal{M}}^{k_{i}}=\left(\mathcal{P}_{k_{i}}^{k_{i}}\right)^{T} \cdot \widehat{\mathcal{H}}^{k_{i}} \cdot \mathcal{K}_{i}
$$

which may be written in matrix form as

is then obtained for the outermost independent generalized accelerations $\dot{\mathbf{u}}_{\mathrm{i}}$.

For the remaining independent coordinates on the loop $\left(k_{i} \leq p_{i}\right)$, the associated quantities and equations of motion are almost those of Section 3, differing only in the definitions of the terms associated with the virtual terminal body $p_{i}$, which are instead

$$
\begin{gathered}
\widehat{\mathcal{I}}^{p_{i} ; 0_{i}}=\breve{\mathcal{I}}^{p_{i} ; 0_{i}}-\sum_{k=p_{i}+1}^{n_{i}}\left(\mathcal{Y}^{k}\right)^{T} \cdot \breve{\mathcal{H}}^{k} \\
\cdot \mathcal{K}_{i}\left(\widehat{\mathcal{M}}_{M}\right)^{-1} \mathcal{K}_{i}^{T} \cdot \widehat{\mathcal{I}}_{M}^{0_{i}}
\end{gathered}
$$

$$
\begin{aligned}
{ }^{0_{i}} \widehat{\mathcal{I}}^{p_{i}}={ }^{0_{i}} \breve{\mathcal{I}}^{p_{i}}-\sum_{k=p_{i}+1}^{n_{i}}\left(\mathcal{Y}^{k}\right)^{T} \cdot \breve{\mathcal{H}}^{k} \\
\cdot \mathcal{K}_{i}\left(\widehat{\mathcal{M}}_{M}\right)^{-1} \mathcal{K}_{i}^{T} \cdot \widehat{\mathcal{I}}_{M}^{p_{i}} \\
\widehat{\mathcal{F}}^{p_{i}}=\breve{\mathcal{F}}^{p_{i}}-\sum_{k=p_{i}+1}^{n_{i}}\left(\mathcal{Y}^{k}\right)^{T} \cdot \breve{\mathcal{H}}^{k} \\
\cdot \mathcal{K}_{i}\left(\widehat{\mathcal{M}}_{M}\right)^{-1} \mathcal{K}_{i}^{T} \cdot \widehat{\mathcal{F}}_{M}
\end{aligned}
$$

And the recursion proceeds exactly as before. 


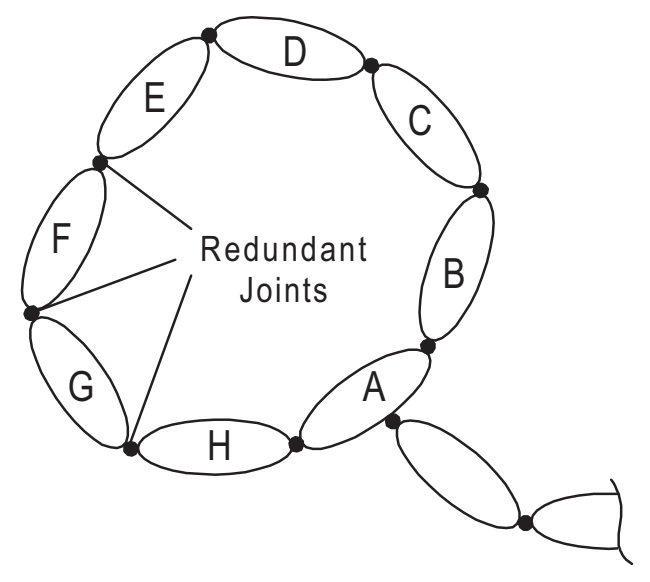

FIGURE 4. Arbitrary singular loop.

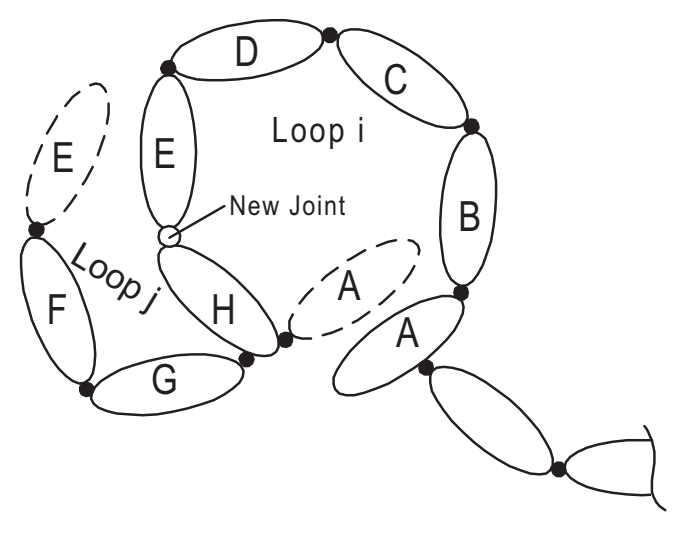

FIGURE 5. Nonsingular representation of the loop of Fig. 4.

\section{GENERAL COUPLED LOOPS}

A second method of avoiding singularities in the RCR formulation involves altering the topology of the multibody system so that valid dependent degrees of freedom are adjacent and at the end of all open loops. The topology adjustment involves the addition of fictitious (although completely valid) joints to the system and relies on the ability to handle systems of coupled loops that do not share a common base body.

An arbitrary closed loop with an invalid set of terminal joints is illustrated in Figure 4. A trivial solution to this problem, which may be valid in many cases, is to simply "work the loop in the opposite direction." Thus in the presence of singular coordinate selection at "both ends," adjusted loops such as that shown in Figure 5 can be used to avoid the singularity.

The computation of two coupled loops $i$ and $j$ with different base bodies $0_{i}$ and $0_{j}$ requires two different representations of the spatial acceleration relative to inertial frame for bodies along the intersection of both loops.

$$
\begin{aligned}
& { }^{N} \widehat{\mathcal{A}}^{k}=\left({ }^{0} \mathcal{S}^{k}\right)^{T} \cdot{ }^{N} \widehat{\mathcal{A}}^{0_{i}}+{ }^{0_{i}} \widehat{\mathcal{A}}^{k} \\
& { }^{N} \widehat{\mathcal{A}}^{k}=\left({ }^{{ }_{j}} \mathcal{S}^{k}\right)^{T} \cdot{ }^{N} \widehat{\mathcal{A}}^{0_{j}}+{ }^{{ }_{j}} \widehat{\mathcal{A}}^{k}
\end{aligned}
$$

Noting the relationships

$$
\begin{aligned}
& { }^{N} \widehat{\mathcal{A}}^{0_{j}}=\left({ }^{0_{i}} \mathcal{S}^{0_{j}}\right)^{T} \cdot{ }^{N} \widehat{\mathcal{A}}^{0_{i}}+{ }^{0_{i}} \widehat{\mathcal{A}}^{0_{j}} \\
& { }^{0_{i}} \widehat{\mathcal{A}}^{k}=\left({ }^{0_{j}} \mathcal{S}^{k}\right)^{T} \cdot{ }^{0_{i}} \widehat{\mathcal{A}}^{0_{j}}+{ }^{0_{j}} \widehat{\mathcal{A}}^{k}
\end{aligned}
$$

substitution and simplification of either results in

$$
{ }^{N} \widehat{\mathcal{A}}^{k}=\left({ }^{0_{i}} \mathcal{S}^{k}\right)^{T} \cdot{ }^{N} \widehat{\mathcal{A}}^{0_{i}}+\left({ }^{0_{j}} \mathcal{S}^{k}\right)^{T} \cdot{ }^{0_{i}} \widehat{\mathcal{A}}^{0_{j}}+{ }^{0_{j}} \widehat{\mathcal{A}}^{k}
$$

Using relationship (107) to handle coupled loops within the RCR is simply a matter of bookkeeping. The local kinematics remain unchanged, and the dynamics of the branching body $b$ from which the two open chains extend are given by

$$
\sum_{k \in \operatorname{Des}[b] \cup b}\left(\tilde{\mathcal{P}}_{b}^{k}\right)^{T} \cdot\left(\mathcal{I}^{k} \cdot{ }^{N} \widehat{\mathcal{A}}^{k}-\mathcal{F}^{k}\right)=0
$$

If the branching body's associated generalized coordinate is an independent coordinate (which it is not in coupled loops resulting from topology "adjustments" for 
the purposes of avoiding a singular coordinate selection), the equation of motion is

$$
\begin{aligned}
& \left(\mathcal{P}_{b}^{b}\right)^{T} \cdot\left(\mathcal{I}^{b} \cdot{ }^{N} \widehat{\mathcal{A}}^{b}-\mathcal{F}^{b}\right) \\
& +\sum_{k \in \operatorname{Des}[b]}\left(\mathcal{P}_{b}^{k}\right)^{T} \cdot\left(\mathcal{I}^{k} \cdot{ }^{N} \widehat{\mathcal{A}}^{k}-\mathcal{F}^{k}\right)=0
\end{aligned}
$$

Substituting from the recursive equation of motion (57) associated with each of the first bodies $\left(f_{i}\right.$ and $\left.f_{j}\right)$ in the two branching "opened" loops, and again assuming that both bodies have associated independent coordinates (which one is not in the case of the topology "adjustment"), one obtains

$$
\begin{aligned}
0 & =\left(\mathcal{P}_{b}^{b}\right)^{T} \cdot\left(\mathcal{I}^{b} \cdot{ }^{N} \widehat{\mathcal{A}}^{b}-\mathcal{F}^{b}\right)+\left(\mathcal{P}_{b}^{b}\right)^{T} \cdot \mathcal{S}^{f_{i}} \\
& \cdot\left(\widehat{\mathcal{I}}^{f_{i} ; 0_{i}} \cdot{ }^{N} \widehat{\mathcal{A}}^{0_{i}}+{ }^{0_{i}} \widehat{\mathcal{I}}^{f_{i}} \cdot{ }^{0_{i}} \widehat{\mathcal{A}}^{f_{i}}-\widehat{\mathcal{F}}^{f_{i}}\right)+\left(\mathcal{P}_{b}^{b}\right)^{T} \cdot \mathcal{S} f^{j} \\
& \cdot\left(\widehat{\mathcal{I}}^{f_{j} ; 0_{j}} \cdot{ }^{N} \widehat{\mathcal{A}}^{0_{j}}+{ }^{0_{j}} \widehat{\mathcal{I}}^{f_{j}} \cdot{ }^{0_{j}} \widehat{\mathcal{A}}^{f_{j}}-\widehat{\mathcal{F}}^{f_{j}}\right)
\end{aligned}
$$

Plugging in the usual recursive relationships (4) for ${ }_{0}{ }_{i} \widehat{\mathcal{A}}^{f_{i}}$ and ${ }^{0_{i}} \widehat{\mathcal{A}}^{f_{j}}$ as well as the form of the solutions for $\dot{u}_{f_{i}}$ and $\dot{u}_{f j}$ given in (59), results in an equation explicit only in contributions from quantities associated with bodies $0_{i}, 0_{j}$, and $b$. However the four spatial inertia coefficients of ${ }^{N} \hat{\mathcal{A}}^{0 i},{ }^{N} \hat{\mathcal{A}}^{0 j},{ }^{0 i} \hat{\mathcal{A}}^{b}$, and ${ }^{0 j} \hat{\mathcal{A}}^{b}$ cannot be used directly to form recursive spatial inertias $\hat{\mathcal{I}}$. Instead the coefficients are combined and distributed as indicated by equations (105) and (106) to produce three coefficients of the spa- tial accelerations ${ }^{N} \hat{\mathcal{A}}^{0 i}$, ${ }^{0 i} \hat{\mathcal{A}}^{0 j}$, and ${ }^{0 j} \hat{\mathcal{A}}^{b}$. And Using (107) to decompose ${ }^{N} \hat{\mathcal{A}}^{b}$ one may write

$$
0=\left(\mathcal{P}_{b}^{b}\right)^{T} \cdot\left(\begin{array}{l}
\widehat{\mathcal{I}}^{b ; 0_{i}} \cdot{ }^{N} \widehat{\mathcal{A}}^{0_{i}}+{ }^{0_{i}} \widehat{\mathcal{I}}^{b ; 0_{j}} \cdot{ }^{0_{i}} \widehat{\mathcal{A}}^{0_{j}} \\
+{ }^{0_{j}} \widehat{\mathcal{I}}^{b} \cdot{ }^{0_{j}} \widehat{\mathcal{A}}^{b}-\widehat{\mathcal{F}}^{b}
\end{array}\right)
$$

The three-term representation of (111) only applies on the bodies which are common to both loops. As on the base body of the inner loop (in this case $j$ ) ${ }^{0 j} \hat{\mathcal{A}}^{k}={ }^{0 j} \hat{\mathcal{A}}^{0 j}=0$, and the reduction "back" to two terms is automatic.

In the case of dependent coordinates being present at the intersection of the two loops, relationship (45) allows the three terms to be constructed at the first independent coordinate inboard of $b$. And solutions involving dependent $f_{i}$ are similarly solved.

\section{DISCUSSION AND RESULTS}

One of the simplest multibody configurations for which the RCR formulation of Section 3 will break down is the planar five-bar linkage represented in Figure 6. In this system, the action of coordinates $q_{1}, q_{2}$, and $q_{3}$ are that of revolute joints, and $q_{4}$ and $q_{5}$ represent the motion of telescoping prismatic joints. It should be clear that when restricted to a forward sweep of the five-bar, the RCR will break down as a result of the invalid selection of dependent coordinates (3-5).

FIGURE 6. Planar five-bar mechanism.

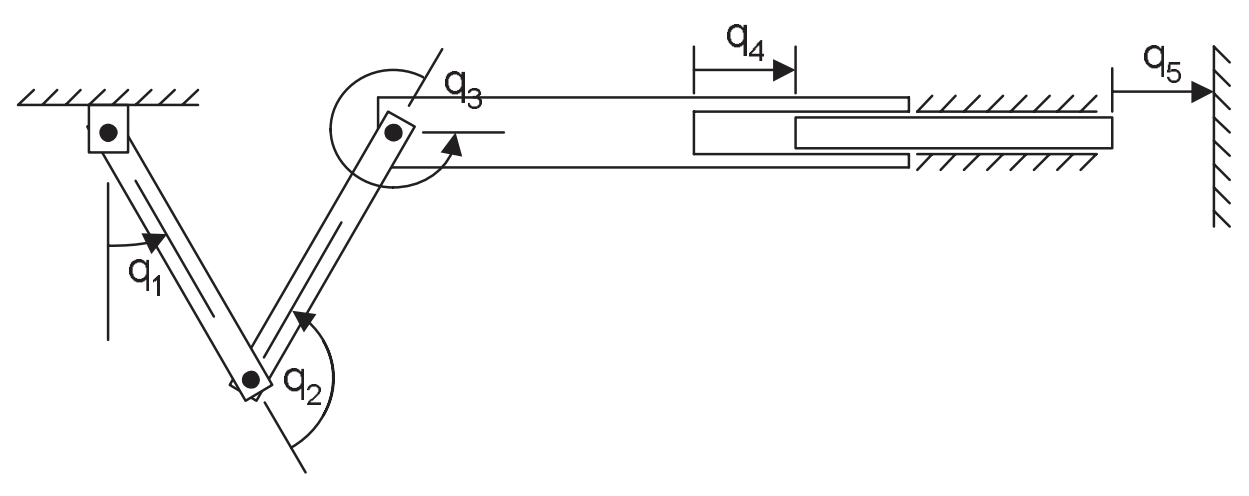




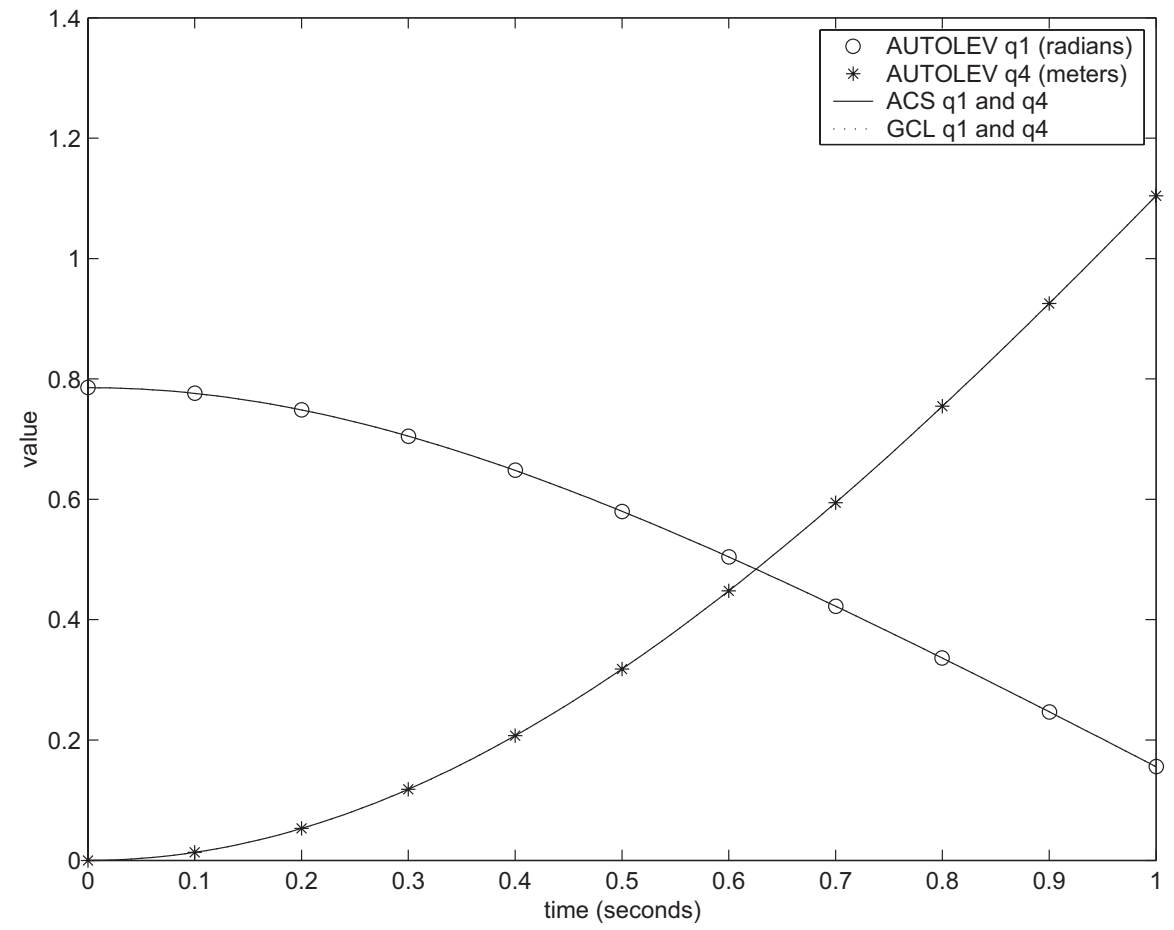

FIGURE 7. FIve-bar simulation results.
The arbitrary coordinate selection (ACS) method of Section 4 does, however, exhibit the expected solution (as obtained with AUTOLEV) as demonstrated by the independent coordinate trajectories shown in Figure
7. In this example, coordinates $q_{2}, q_{3}$, and $q_{5}$ are used as the dependent coordinates, all bars are thin rods of unit length (meters) and mass (kilograms), and the linkage is released from rest in the vertical plane with
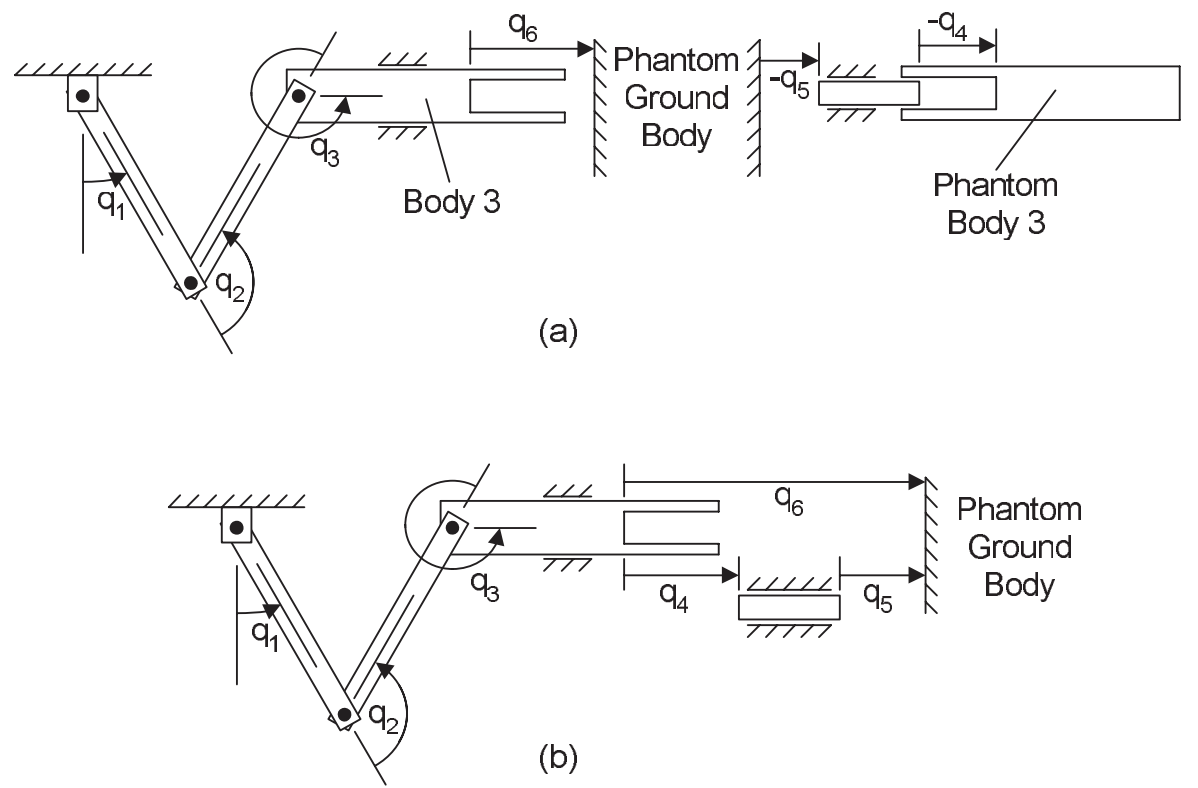

FIGURE 8. Available five-bar tree topologies. 
the initial conditions $q_{1}=\frac{\pi}{4}, q_{2}=\frac{\pi}{2}, q_{3}=-\frac{\pi}{2}, q_{4}=0$, $\left.q_{5}=0\right)$.

The general coupled loop (GCL) solution of Section 5 for the same five-bar mechanism is also shown in Figure 7 (the motions are identical). In the case of this simple mechanism, the two multibody tree topologies of Figure 8 are possible to avoid the singularity. It should be noted that the system of Figure $8 \mathrm{~b}$ is actually an existing solution of a type demonstrated in Anderson and Critchley [23] (e.g., coupled loops with the same base body), the form of which is only available for planar and some special spatial loops. For this reason only the solution indicated by Figure 8a corresponding to the general coupled loop procedure is given in Figure 7.

Although each of the two methods discussed in Sections 4 and 5 obtain the same result, the computational order of the algorithms is very different. The complexity of the arbitrary coordinate selection method is a cubic function of the number of independent coordinates $n_{I}$ located in between the dependent ones $\left(p_{i}+1\right.$ and $n_{i}$ ), owing to the inversion of $\widehat{\mathcal{M}}_{M}$. In this respect, $n_{I}$ is bounded only by the total number of independent coordinates participating in a given loop $\left(n_{i}-m_{i}-1\right)$ and should therefore be treated as a loop local $O\left(n_{i}{ }^{3}\right)$ operation. This worst case behavior can best be observed in the case of spatial loops with large planar portions adjacent to the loop base body.

The additional complexity of the coupled loop solution, if applied intelligently, never exceeds $O\left(m_{i}\right)$. The low order resulting from the addition of multiple loops that may be required to avoid several singularities is obtained because the newly formed loops need never be coupled to each other. Hence, the worst case behavior is an $m_{i}$ coordinate growth in the description of the loop, which is solved in linear time $O\left(n+2 m_{i}\right)$ or simply $O(n+m)$.

The coupled loop solution also represents the last piece of a generalized recursive coordinate reduction (GRCR) method which yields $O(n+m)$ performance for arbitrary multibody system configurations. As such, the GRCR should be used to replace the traditional "order $n$ " constraint formulation in any application save those where the constraint forces are both explicitly required and few in number. That is, the constraint forces can be extracted from the GRCR in the usual way, which may be less efficient only in the case of very lightly constrained systems.

It should be noted that in the presence of heavy local loop coupling, such as one would find in a multibody lattice (or mesh), the local coupling will produce $n_{b}$ spatial inertia terms on each body in a local loop. The computational complexity of such methods in general is unclear, because optimal tree representations of such systems are required. In some cases the results can still be obtained in $O(n+m)$, in others $O\left(n+n_{b} m\right)$ is more appropriate, while $O\left(\log _{n b} n\right)$ may be an appropriate upper bound. The application of RCR methods to such systems is a topic of current and future research.

The GRCR presented in this paper can also be exploited to obtain a parallel computation algorithm with logarithmic time complexity $\left(O\left(\log _{k} n\right)\right)$. This forthcoming work promises a theoretical minimum order of computations $(O(n))$ on the theoretical minimum order of processors $(O(n))$ as well as increased flexibility and accuracy as a result of the form of the constraint treatment.

\section{CONCLUSIONS}

The recursive coordinate reduction method which has previously been shown to offer true $O(n+m)$ performance for a large class of multibody systems has been expanded to accommodate the complete set of multibody systems. Two methods have been derived to cope with singularities in the original formulation resulting from the fixed selection of dependent coordinates. The arbitrary coordinate selection method requiring the inverse of a $n_{I} \times n_{I}$ matrix is locally cubic, $O\left(n_{I}^{3}\right)$, and is superior to the traditional $O(n)$ closed-loop solution if the system loops are small or the coordinates need only be altered slightly.

The other solution involving the creation of a coupled loop representation of the singular loop offers $O(n+m)$ complexity. The fully recursive general coupled loop solution presented also completes a generalized recursive coordinate reduction method that can be applied to arbitrary multibody systems resulting in $O(n+m)$ performance for all but the most heavily coupled multibody latices. As such, this method should be used in place of the traditional $O(n)$ constraint method for superior computational performance.

\section{ACKNOWLEDGMENT}

Support for this work received under National Science Foundation through award No. 9733684 is gratefully acknowledged. 


\section{REFERENCES}

1. R.Featherstone. The calculation of robotic dynamics using articulated body inertias. International Journal of Robotics Research, 2(1):13-30, 1983.

2. A. F. Vereshchagin. Gauss principle of least constraint for modeling the dynamics of automatic manipulators using a digital computer. Soviet Physics [Doklady], 20(1):33-34, 1975.

3. W. W. Armstrong. Recursive solution to the equations of motion of an $n$-link manipulator. In: Proceedings of the Fifth World Congress on the Theory of Machines and Mechanisms, volume 2, 1342-1346, 1979.

4. J. S. Y. Luh, M. W. Walker, R. P. C. Paul. On-line computational scheme for mechanical manipulators. Journal of Dynamic Systems, Measurements, and Controls, 102:69-76, 1980.

5. M. W. Walker, D. E. Orin. Efficient dynamic computer simulation of robotic mechanisms. Journal of Dynamic Systems, Measurements, and Controls, 104: 205-211, 1982.

6. J. M. Hollerbach. A recursive Lagrangian formulation of manipulator dynamics and a comparative study of dynamics formulation complexity. IEEE Transactions on Systems, Man, and Cybernetics, SMC10(11):730-736, 1980.

7. A.K. Banerjee. Block-diagonal equations for multibody elastodynamics with geometric stiffness and constraints. Journal of Guidance, Control, and Dynamics, 16(6):1092-1100, 1993.

8. A. Jain, G. Rodriguez. Recursive flexible multibody system dynamics using spatial operators. Journal of Guidance, Control, and Dynamics, 15(6):1453-1466, 1992.

9. K. S. Anderson. An efficient formulation for the modeling of general multi-flexible-body constrained systems. International Journal of Solids Structures, 30(7):921-945, 1993.

10. G. Rodriguez. Kalman filtering, smoothing, and recursive robot arm forward and inverse dynamics. IEEE Journal of Robotics and Automatation, RA-3(6): 624-639, 1987.

11. K. Kreutz-Delgado, A.Jain, G. Rodriguez. Recursive formulation of operational space control. International Journal of Robotics Research, 11(4):320-328, 1992.

12. K. S. Anderson, Y. H. Hsu. Analytic full-recursive sensitivity analysis for multibody dynamic chain systems. Multibody Systems Dynamics, 8(1):1-27, 2002.

13. Y. H. Hsu, K. S. Anderson. Recursive sensitivity analysis for constrained multi-rigid-body dynamic systems design optimization. Structural and Multidisciplinary Optimization, 24(4):312-324, 2002.

14. D. Bae, J. G. Kuhl, E. J. Haug. A recursive formation for constrained mechanical system dynamics: Part III, Parallel processing implementation. Mechanisms, Structures, and Machines, 16:249-269, 1988.

15. K.S.Anderson. An efficient modeling of constrained multibody systems for application with parallel computing. Zeitschrift Angewandte Mathematik und Mechanik, 73(6):935-939, 1993.

16. K.S.Anderson, S. Duan. A hybrid parallelizable low order algorithm for dynamics of multi-rigid-body systems: Part I, Chain systems. Mathematical and Computer Modeling, 30:193-215, 1999.

17. R. Featherstone. A divide-and-conquer articulated body algorithm for parallel calculation of rigid body dynamics. Part 1: Basic algorithm. International Journal of Robotics Research, 18(9):867-875, 1999.

18. R. Featherstone. Robot Dynamics Algorithms. Kluwer, New York, 1987.

19. D. S. Bae, E. J. Haug. A recursive formation for constrained mechanical system dynamics: Part II, Closed loop systems. Mechanisms, Structures, and Machines, 15(4):481-506, 1987.

20. V. Stejskal, M. Valášek. Kinematics and Dynamics of Machinery. Marcel Dekker, New York, 1996.

21. K.S.Anderson. Improved order- $n$ performance algorithm for the simulation of constrained multi-rigidbody systems. In: Proceedings of the Third Symposium on Multibody Dynamics and Vibrations, ASMEDesign Engineering Technical Conference 2001, (DETC01), DETC2001/VIB-21334, Pittsburgh, PA, 2001.

22. K. S. Anderson. An order- $n$ formulation for motion simulation of general constrained multi-rigid-body systems. Computers and Structures, 43(3):565-572, 1992.

23. K. S. Anderson, J. H. Critchley. Improved order$N$ performance algorithm for the simulation of constrained multi-rigid-body systems. Multibody Systems Dynamics, 9(2):185-212, 2003

24. T. R. Kane, D. A. Levinson. Dynamics: Theory and Application. McGraw-Hill, New York, 1985.

25. P. C. Mitiguy, T. R. Kane. Motion variables leading to efficient equations of motion. International Journal of Robotics Research, 15(5):522-532, 1996. 